Supporting Information

For the article entitled

\title{
Iridium-Catalyzed Cross-Coupling Reactions of Alkenes by Hydrogen Transfer
}

Keke Meng, Yaling Sun, Jian Zhang,* Kaiyun Zhang, Xiaohui Ji, Liyuan Ding, and Guofu Zhong*

Email: zhangjian@hznu.edu.cn; zgf@hznu.edu.cn

\section{Table of Contents}

General Methods

General Procedure for Catalytic Cross-Coupling Reactions $\quad$ SI-5

$\begin{array}{ll}\text { Characterization of Butadienes } & \text { SI-5 }\end{array}$

$\begin{array}{ll}\text { Deuterium-Labeled Experiments } & \text { SI-11 }\end{array}$

\begin{tabular}{l|l} 
KIE Experiments & SI-14
\end{tabular}

$\begin{array}{ll}\text { Competition Reaction } & \text { SI-16 }\end{array}$

$\begin{array}{ll}\text { Hammett-Plot with Analysis } & \text { SI-17 }\end{array}$

$\begin{array}{lr}\text { Synthetic Application } & \text { SI-18 }\end{array}$

$\begin{array}{ll}\text { References } & \text { SI-20 }\end{array}$

\begin{tabular}{l|l} 
NMR Spectra & SI-21
\end{tabular} 


\section{General Methods}

Analytical thin layer chromatography (TLC) was performed using Merck 60 F254 precoated silica gel plate $(0.2 \mathrm{~mm}$ thickness). Subsequent to elution, plates were visualized using UV radiation $(254 \mathrm{~nm})$ on Spectroline Model ENF-24061/F254 nm. Further visualization was possible by staining with basic solution of potassium permanganate or acidic solution of ceric molybdate. Flash column chromatography was performed using Merck aluminium oxide 90 active neutral with freshly distilled solvents. Columns were typically packed as slurry and equilibrated with the appropriate solvent system prior to use. Proton nuclear magnetic resonance spectra $\left({ }^{1} \mathrm{H}\right.$ NMR) were recorded on Bruker AMX400 and $500 \mathrm{MHz}$ spectrophotometer $\left(\mathrm{CDCl}_{3}\right.$ as solvent). Chemical shifts for ${ }^{1} \mathrm{H}$ NMR spectra are reported as $\delta$ in units of parts per million (ppm) downfield from $\operatorname{SiMe}_{4}(\delta 0.0)$ and relative to the signal of chloroform- $d(\delta$ 7.26, singlet). Multiplicities were given as: s (singlet), $\mathrm{d}$ (doublet), $\mathrm{t}$ (triplet), dd (doublets of doublet) or $\mathrm{m}$ (multiplets). The number of protons ( $\mathrm{n}$ ) for a given resonance is indicated by nH. Coupling constants are reported as a $J$ value in Hz. Carbon nuclear magnetic resonance spectra $\left({ }^{13} \mathrm{C}\right.$ NMR) are reported as $\delta$ in units of parts per million (ppm) downfield from $\mathrm{SiMe}_{4}(\delta 0.0)$ and relative to the signal of chloroform- $d(\delta 77.0$, triplet). Mass spectrometry was performed by Waters Q-Tof Premier Micromass instrument, using Electro Spray Ionization (ESI) mode. IR spectra were recorded as thin films on $\mathrm{KBr}$ or $\mathrm{NaCl}$ plates on a Bio-Rad FTS 165 FTIR spectrometer and are reported in frequency of absorption $\left(\mathrm{cm}^{-1}\right)$. $\left[\operatorname{IrCp}^{*} \mathrm{Cl}_{2}\right]_{2},[\operatorname{Ir}(\mathrm{cod}) \mathrm{Cl}]_{2}$ and $\mathrm{AgBF}_{4}$ were purchased from Energy and used directly. Other reagents, unless otherwise noted below, are commercially available from Alfa Aesar (China) Chemical Co., Ltd. and used without further purification. Acrylamides were prepared by reported methods. ${ }^{1-2}$ 


\section{General Procedure for Ir-Catalyzed Alkene-Alkene Cross-Coupling}<smiles>[R]C=C[NH2+][CH]C([R])=C([R])[R]</smiles>

4

5
$\left[\mathrm{IrCp}{ }^{*} \mathrm{Cl}_{2}\right]_{2}(10 \mathrm{~mol} \%)$

$\mathrm{AgBF}_{4}(50 \mathrm{~mol} \%)$

ethyl acetate $(0.2 \mathrm{M})$ $100^{\circ} \mathrm{C}, 6 \mathrm{~h}$<smiles>[R]C=CC([R])=C([R])C(=O)N([R])[R]</smiles>

6

An oven-dried vial was charged with $\left[\operatorname{IrCp}^{*} \mathrm{Cl}_{2}\right]_{2}(15.9 \mathrm{mg}, 10 \mathrm{~mol} \%), \mathrm{AgBF}_{4}(19.5 \mathrm{mg}, 50 \mathrm{~mol} \%)$ and EtOAc ( $1 \mathrm{~mL})$. Then, alkene 2 ( $0.4 \mathrm{mmol}, 2.0$ equiv) and acrylamide $\mathbf{1}(0.2 \mathrm{mmol}, 1.0$ equiv) were added into the solution in sequence. The vial was sealed under argon and heated to $100{ }^{\circ} \mathrm{C}$ with stirring for 6 hours. After cooling down, the mixture was concentrated to give the crude product which was directly applied to a flash column chromatography for purification ( $\mathrm{MeOH} / \mathrm{CH}_{2} \mathrm{Cl}_{2}$ mixtures).

\section{Characterization of Butadienes}<smiles>CCOP(=O)(/C=C/C=C(/C)C(=O)N(C)C)OCC</smiles>

Diethyl((1E,3Z)-5-(dimethylamino)-4-methyl-5-oxopenta-1,3-dien-1-yl) phosphonate (3aa). Following the general experiment procedure, 3aa was obtained as a yellow liquid $(86 \%, 48 \mathrm{mg}) .{ }^{1} \mathrm{H}$ NMR $\left(500 \mathrm{MHz}, \mathrm{CDCl}_{3}\right): \delta$ $=6.81-6.90(\mathrm{~m}, 1 \mathrm{H}), 6.00(\mathrm{~d}, J=11.0 \mathrm{~Hz}, 1 \mathrm{H}), 5.63(\mathrm{dd}, J=17.0 \mathrm{~Hz}, J=18.5 \mathrm{~Hz}, 1 \mathrm{H}), 3.96-4.02(\mathrm{~m}$, 4H), 2.96 (s, 3H), 2.89 (s, 3H ), 1.97 (s, 3H), 1.24 (t, $J=7.0 \mathrm{~Hz}, 6 \mathrm{H}) \cdot{ }^{13} \mathrm{C}$ NMR $\left(125 \mathrm{MHz}, \mathrm{CDCl}_{3}\right): \delta=$ $170.22,143.60\left(\mathrm{~d}, J_{\mathrm{C}-\mathrm{P}}=6.3 \mathrm{~Hz}\right), 142.14,126.86\left(\mathrm{~d}, J_{\mathrm{C}-\mathrm{P}}=27.5 \mathrm{~Hz}\right), 118.59\left(\mathrm{~d}, J_{\mathrm{C}-\mathrm{P}}=190.0 \mathrm{~Hz}\right), 61.89$, 61.85, 37.73, 34.33, 20.95, 16.41, 16.36. HR-MS (ESI): $\mathrm{m} / \mathrm{z}$ calculated for $\mathrm{C}_{12} \mathrm{H}_{22} \mathrm{NO}_{4} \mathrm{P}:[\mathrm{M}+\mathrm{H}]^{+}$: 276.1359, found: 276.1357. FTIR (KBr, $\left.\mathrm{cm}^{-1}\right): 3507.37,3444.67,3417.83,2462.83,1651.43,1633.68$, 1614.85, 1384.38.<smiles>CCOP(=O)(/C=C/C=C(\C(=O)N(C)C)c1ccccc1)OCC</smiles>

\section{Diethyl((1E,3Z)-5-(dimethylamino)-5-oxo-4-phenylpenta-1,3-dien-1} -yl) phosphonate (3ba).

Following the general experiment procedure, 3ba was obtained as a yellow liquid $(83 \%, 68 \mathrm{mg}) .{ }^{1} \mathrm{H}$ NMR $\left(500 \mathrm{MHz}, \mathrm{CDCl}_{3}\right): \delta=7.35-7.45(\mathrm{~m}, 5 \mathrm{H}), 7.08-7.18(\mathrm{~m}$, 1H), $6.69(\mathrm{~d}, J=12.0 \mathrm{~Hz}, 1 \mathrm{H}), 5.93(\mathrm{dd}, J=17.0 \mathrm{~Hz}, J=18.5 \mathrm{~Hz}, 1 \mathrm{H}), 4.07-4.13(\mathrm{~m}, 4 \mathrm{H}), 3.14(\mathrm{~s}, 3 \mathrm{H})$, $2.88(\mathrm{~s}, 3 \mathrm{H}), 1.34$ (t, $J=7.0 \mathrm{~Hz}, 6 \mathrm{H}) .{ }^{13} \mathrm{C}$ NMR $\left(125 \mathrm{MHz}, \mathrm{CDCl}_{3}\right): \delta=168.51,144.48,143.59\left(\mathrm{~d}, J_{\mathrm{C}-\mathrm{P}}\right.$ $=6.3 \mathrm{~Hz}), 134.55,129.55,129.16,126.10,124.99\left(\mathrm{~d}, J_{\mathrm{C}-\mathrm{P}}=27.5 \mathrm{~Hz}\right), 120.82\left(\mathrm{~d}, J_{\mathrm{C}-\mathrm{P}}=190.0 \mathrm{~Hz}\right)$, 
62.02, 61.98, 38.00, 34.56, 16.47, 16.42. HR-MS (ESI): $\mathrm{m} / \mathrm{z}$ calculated for $\mathrm{C}_{17} \mathrm{H}_{24} \mathrm{NO}_{4} \mathrm{P}:[\mathrm{M}+\mathrm{H}]^{+}$: 338.1516, found: 338.1511. FTIR (KBr, $\left.\mathrm{cm}^{-1}\right)$ : 3472.93, 3444.65, 3423.89, 2355.67, 1651.30, 1384.31, 1025.76.<smiles>CCOP(=O)(/C=C/C=C(/C(=O)N(C)C)c1ccc(Cl)cc1)OCC</smiles>

\section{Diethyl((1E,3Z)-4-(4-chlorophenyl)-5-(dimethylamino)-5-oxop} enta-1,3-dien-1-yl)phosphonate (3ca).

Following the general experiment procedure, 3 ca was obtained as a yellow liquid $(61 \%, 45 \mathrm{mg}) .{ }^{1} \mathrm{H}$ NMR $\left(500 \mathrm{MHz}, \mathrm{CDCl}_{3}\right): \delta=7.34-7.40(\mathrm{~m}, 4 \mathrm{H}), 7.06-7.16(\mathrm{~m}$, 1H), $6.67(\mathrm{~d}, J=11.5 \mathrm{~Hz}, 1 \mathrm{H}), 5.95(\mathrm{dd}, J=17.0 \mathrm{~Hz}, J=18.0 \mathrm{~Hz}, 1 \mathrm{H}), 4.07-4.13(\mathrm{~m}, 4 \mathrm{H}), 3.14(\mathrm{~s}, 3 \mathrm{H})$ $2.87(\mathrm{~s}, 3 \mathrm{H}), 1.34(\mathrm{t}, J=7.0 \mathrm{~Hz}, 6 \mathrm{H}) .{ }^{13} \mathrm{C} \mathrm{NMR}\left(125 \mathrm{MHz}, \mathrm{CDCl}_{3}\right): \delta=168.10,143.23\left(\mathrm{~d}, J_{\mathrm{C}-\mathrm{P}}=7.5\right.$ $\mathrm{Hz}), 143.15,135.49,133.10,129.41,127.39,125.40$ (d, $\left.J_{\mathrm{C}-\mathrm{P}}=27.5 \mathrm{~Hz}\right), 121.52\left(\mathrm{~d}, J_{\mathrm{C}-\mathrm{P}}=188.8 \mathrm{~Hz}\right)$, 62.07, 62.03, 37.97, 34.60, 16.48, 16.43. HR-MS (ESI): $\mathrm{m} / \mathrm{z}$ calculated for $\mathrm{C}_{17} \mathrm{H}_{23} \mathrm{ClNO}_{4} \mathrm{P}:[\mathrm{M}+\mathrm{H}]^{+}$: 372.1126, found: 372.1119. FTIR (KBr, $\left.\mathrm{cm}^{-1}\right): 3646.11,3626.62,3453.43,2378.78,1651.17,1633.95$, $1384.55,1050.32,1024.91$<smiles>CCOP(=O)(/C=C/C=C(/C(=O)N(C)C)c1ccc(F)cc1)OCC</smiles>

Diethyl((1E,3Z)-5-(dimethylamino)-4-(4-fluorophenyl)-5-oxope nta-1,3-dien-1-yl)phosphonate (3da).

Following the general experiment procedure, $\mathbf{3 d a}$ was obtained as a yellow liquid $(85 \%, 61 \mathrm{mg}) .{ }^{1} \mathrm{H}$ NMR $\left(500 \mathrm{MHz}, \mathrm{CDCl}_{3}\right): \delta=7.35-7.37(\mathrm{~m}, 2 \mathrm{H}), 6.98-7.05(\mathrm{~m}$, 3H), $6.55(\mathrm{~d}, J=11.5 \mathrm{~Hz}, 1 \mathrm{H}), 5.86(\mathrm{dd}, J=16.0 \mathrm{~Hz}, J=18.5 \mathrm{~Hz}, 1 \mathrm{H}), 3.99-4.05(\mathrm{~m}, 4 \mathrm{H}), 3.06(\mathrm{~s}, 3 \mathrm{H})$ $2.80(\mathrm{~s}, 3 \mathrm{H}), 1.26(\mathrm{t}, J=7.0 \mathrm{~Hz}, 6 \mathrm{H}) .{ }^{13} \mathrm{C} \mathrm{NMR}\left(125 \mathrm{MHz}, \mathrm{CDCl}_{3}\right): \delta=167.26,162.40\left(\mathrm{~d}, J_{\mathrm{C}-\mathrm{F}}=248.8\right.$ $\mathrm{Hz}), 142.82\left(\mathrm{~d}, J_{\mathrm{C}-\mathrm{P}}=6.3 \mathrm{~Hz}\right), 142.22,129.78\left(\mathrm{q}, J_{\mathrm{C}-\mathrm{F}}=1.25 \mathrm{~Hz}\right), 127.00\left(\mathrm{~d}, J_{\mathrm{C}-\mathrm{F}}=8.8 \mathrm{~Hz}\right), 123.79(\mathrm{dd}$, $\left.J_{\mathrm{C}-\mathrm{P}}=1.3 \mathrm{~Hz}, J_{\mathrm{C}-\mathrm{P}}=27.5 \mathrm{~Hz}\right), 119.99\left(\mathrm{~d}, J_{\mathrm{C}-\mathrm{P}}=190.0 \mathrm{~Hz}\right), 115.21\left(\mathrm{~d}, J_{\mathrm{C}-\mathrm{F}}=22.5 \mathrm{~Hz}\right), 61.01,60.97$, 36.91, 33.53, 15.43, 15.38. HR-MS (ESI): $\mathrm{m} / \mathrm{z}$ calculated for $\mathrm{C}_{17} \mathrm{H}_{23} \mathrm{FNO}_{4} \mathrm{P}:[\mathrm{M}+\mathrm{H}]^{+}:$356.1421, found: 356.1419. FTIR $\left(\mathrm{KBr}, \mathrm{cm}^{-1}\right): 3564.40,3472.97,3417.77,1651.38,1621.66,1505.08,1237.54$, 1026.02<smiles>CCOP(=O)(/C=C/C=C(/C(=O)N(C)C)c1ccc(OC)cc1)OCC</smiles>

Diethyl((1E,3Z)-5-(dimethylamino)-4-(4-methoxyphenyl)-5oxopenta-1,3-dien-1-yl)phosphonate (3ea). 
Following the general experiment procedure, 3ea was obtained as a yellow liquid $(60 \%, 44 \mathrm{mg}) .{ }^{1} \mathrm{H}$ $\operatorname{NMR}\left(500 \mathrm{MHz}, \mathrm{CDCl}_{3}\right): \delta=7.37-7.39(\mathrm{~m}, 2 \mathrm{H}), 7.06-7.15(\mathrm{~m}, 1 \mathrm{H}), 6.89-6.91(\mathrm{~m}, 2 \mathrm{H}), 6.60(\mathrm{~d}, J=$ $11.5 \mathrm{~Hz}, 1 \mathrm{H}), 5.87$ (dd, $J=17.0 \mathrm{~Hz}, J=18.5 \mathrm{~Hz}, 1 \mathrm{H}), 4.06-4.12(\mathrm{~m}, 4 \mathrm{H}), 3.83(\mathrm{~s}, 3 \mathrm{H}), 3.14(\mathrm{~s}, 3 \mathrm{H})$, $2.88(\mathrm{~s}, 3 \mathrm{H}), 1.33(\mathrm{t}, J=7.0 \mathrm{~Hz}, 6 \mathrm{H}) .{ }^{13} \mathrm{C} \mathrm{NMR}\left(125 \mathrm{MHz}, \mathrm{CDCl}_{3}\right): \delta=168.84,160.78,144.14,143.99$ $\left(\mathrm{d}, J_{\mathrm{C}-\mathrm{P}}=6.3 \mathrm{~Hz}\right), 127.63,126.97,122.94\left(\mathrm{~d}, J_{\mathrm{C}-\mathrm{P}}=27.5 \mathrm{~Hz}\right), 118.42\left(\mathrm{~d}, J_{\mathrm{C}-\mathrm{P}}=190.0 \mathrm{~Hz}\right), 114.61,62.02$, 61.98, 55.49, 38.04, 34.57, 16.51, 16.46. HR-MS (ESI): $\mathrm{m} / \mathrm{z}$ calculated for $\mathrm{C}_{18} \mathrm{H}_{26} \mathrm{NO}_{5} \mathrm{P}:[\mathrm{M}+\mathrm{H}]^{+}$: 368.1621, found: 368.1616. FTIR (KBr, $\left.\mathrm{cm}^{-1}\right)$ : 3585.30, 3472.93, 3444.80, 2346.56, 1633.83, 1598.26, $1252.64,1025.70$.<smiles>CCOP(=O)(/C=C/C=C(/C(=O)N(C)C)c1ccc(C(F)(F)F)cc1)OCC</smiles>

Diethyl((1E,3Z)-5-(dimethylamino)-5-0xo-4-(4-(trifluoromet hyl)phenyl)penta-1,3-dien-1-yl)phosphonate (3fa).

Following the general experiment procedure, 3 fa was obtained as a yellow liquid $(47 \%, 38 \mathrm{mg}) .{ }^{1} \mathrm{H} \mathrm{NMR}\left(500 \mathrm{MHz}, \mathrm{CDCl}_{3}\right): \delta=7.56-7.64(\mathrm{~m}, 4 \mathrm{H}), 7.08-7.18(\mathrm{~m}$, 1H), $6.74(\mathrm{~d}, J=11.0 \mathrm{~Hz}, 1 \mathrm{H}), 6.01(\mathrm{t}, J=17.5 \mathrm{~Hz}, 1 \mathrm{H}), 4.08-4.14(\mathrm{~m}, 4 \mathrm{H}), 3.15(\mathrm{~s}, 3 \mathrm{H}), 2.88(\mathrm{~s}, 3 \mathrm{H})$, $1.34(\mathrm{t}, J=6.5 \mathrm{~Hz}, 6 \mathrm{H}) .{ }^{13} \mathrm{C} \mathrm{NMR}\left(125 \mathrm{MHz}, \mathrm{CDCl}_{3}\right): \delta=167.84,142.91,142.86,138.27,131.07$ (q, $\left.J_{\mathrm{C}-\mathrm{F}}=32.5 \mathrm{~Hz}\right), 127.12\left(\mathrm{~d}, J_{\mathrm{C}-\mathrm{P}}=27.5 \mathrm{~Hz}\right), 126.48,126.17\left(\mathrm{q}, J_{\mathrm{C}-\mathrm{F}}=2.5 \mathrm{~Hz}\right), 124.31\left(\mathrm{~d}, J_{\mathrm{C}-\mathrm{F}}=270.0 \mathrm{~Hz}\right)$, $122.47\left(\mathrm{~d}, J_{\mathrm{C}-\mathrm{F}}=188.8 \mathrm{~Hz}\right), 62.19,62.14,38.00,34.69,16.52,16.47 . \mathrm{HR}-\mathrm{MS}(\mathrm{ESI}): \mathrm{m} / \mathrm{z}$ calculated for $\mathrm{C}_{18} \mathrm{H}_{23} \mathrm{~F}_{3} \mathrm{NO}_{4} \mathrm{P}:[\mathrm{M}+\mathrm{H}]^{+}:$406.1390, found: 406.1380. FTIR $\left(\mathrm{KBr}, \mathrm{cm}^{-1}\right): 3472.94,3417.89,2924.85$, $1633.96,1434.88,1325.08,1246.19,1026.82,966.23,843.67$.<smiles>CCOP(=O)(/C=C/C=C(/C(=O)N(C)C)c1cccc2ccccc12)OCC</smiles>

Diethyl((1E,3Z)-5-(dimethylamino)-4-(naphthalen-1-yl)-5-oxopenta -1,3-dien-1-yl)phosphonate (3ga).

Following the general experiment procedure, 3ga was obtained as a yellow liquid $(70 \%, 54 \mathrm{mg}) .{ }^{1} \mathrm{H}$ NMR $\left(500 \mathrm{MHz}, \mathrm{CDCl}_{3}\right): \delta=$ 8.29-8.31 (d, $J=8.0 \mathrm{~Hz}, 1 \mathrm{H}), 7.83-7.89(\mathrm{~m}, 2 \mathrm{H}), 7.50-7.59(\mathrm{~m}, 3 \mathrm{H})$, 7.44-7.47 (m, 1H), 7.32-7.40 (m, 1H), $6.59(\mathrm{~d}, J=11.5 \mathrm{~Hz}, 1 \mathrm{H}), 5.93(\mathrm{dd}, J=16.5 \mathrm{~Hz}, J=18.5 \mathrm{~Hz}, 1 \mathrm{H}), 4.11-4.17(\mathrm{~m}, 4 \mathrm{H}), 3.05(\mathrm{~s}, 3 \mathrm{H})$, $2.87(\mathrm{~s}, 3 \mathrm{H}), 1.36(\mathrm{t}, J=7.0 \mathrm{~Hz}, 6 \mathrm{H}) .{ }^{13} \mathrm{C} \mathrm{NMR}\left(125 \mathrm{MHz}, \mathrm{CDCl}_{3}\right): \delta=168.70,143.86,143.66\left(\mathrm{~d}, J_{\mathrm{C}-\mathrm{P}}\right.$ $=6.3 \mathrm{~Hz}), 134.27,134.04,132.25\left(\mathrm{~d}, J_{\mathrm{C}-\mathrm{P}}=27.5 \mathrm{~Hz}\right), 130.65,129.49,128.94,127.02,126.37,126.21$, 125.31, 124.90, $120.26\left(\mathrm{~d}, J_{\mathrm{C}-\mathrm{P}}=188.8 \mathrm{~Hz}\right), 62.14,62.09,37.99,35.10,16.50,16.45 . \mathrm{HR}-\mathrm{MS}$ (ESI): 
$\mathrm{m} / \mathrm{z}$ calculated for $\mathrm{C}_{21} \mathrm{H}_{26} \mathrm{NO}_{4} \mathrm{P}:[\mathrm{M}+\mathrm{H}]^{+}: 388.1672$, found: 388.1666 . FTIR $\left(\mathrm{KBr}, \mathrm{cm}^{-1}\right)$ : 3564.35 , $3444.60,2924.63,1633.48,1470.53,1385.02,1247.67,1024.08,779.43,737.18$.<smiles>CCOP(=O)(/C=C/C1=C(C(=O)N(C)C)CCCC1)OCC</smiles>

\section{Diethyl(E)-(2-(2-(dimethylcarbamoyl)cyclohex-1-en-1-yl)vinyl)phosphon}

ate (3ha). Following the general experiment procedure, 3ha was obtained as a yellow liquid $(83 \%, 52 \mathrm{mg}) .{ }^{1} \mathrm{H} \mathrm{NMR}\left(500 \mathrm{MHz}, \mathrm{CDCl}_{3}\right): \delta=6.95(\mathrm{dd}$, $J=17.0 \mathrm{~Hz}, J=22.0 \mathrm{~Hz}, 1 \mathrm{H}), 5.64(\mathrm{t}, J=17.5 \mathrm{~Hz}, 1 \mathrm{H}), 3.99-4.04(\mathrm{~m}, 4 \mathrm{H})$, $2.97(\mathrm{~s}, 3 \mathrm{H}), 2.86(\mathrm{~s}, 3 \mathrm{H}), 2.42-2.45(\mathrm{~m}, 1 \mathrm{H}), 2.25(\mathrm{~s}, 1 \mathrm{H}), 2.13(\mathrm{~s}, 2 \mathrm{H}), 1.65-1.68(\mathrm{~m}, 4 \mathrm{H}), 1.24(\mathrm{t}, J=$ $6.5 \mathrm{~Hz}, 6 \mathrm{H}) .{ }^{13} \mathrm{C}$ NMR $\left(125 \mathrm{MHz}, \mathrm{CDCl}_{3}\right): \delta=171.28,145.78\left(\mathrm{~d}, J_{\mathrm{C}-\mathrm{P}}=7.5 \mathrm{~Hz}\right), 141.42,130.30\left(\mathrm{~d}, J_{\mathrm{C}-\mathrm{P}}\right.$ $=23.8 \mathrm{~Hz}), 113.59\left(\mathrm{~d}, J_{\mathrm{C}-\mathrm{P}}=65.0 \mathrm{~Hz}\right), 61.87,60.45,37.85,34.35,27.80,23.84,21.71,21.66,16.42$, 16.37. HR-MS (ESI): $\mathrm{m} / \mathrm{z}$ calculated for $\mathrm{C}_{15} \mathrm{H}_{26} \mathrm{NO}_{4} \mathrm{P}:[\mathrm{M}+\mathrm{H}]^{+}:$316.1672, found: 316.1676 . FTIR (KBr, $\left.\mathrm{cm}^{-1}\right): 3646.20,3444.55,2924.94,1660.49,1621.73,1505.00,1384.48,1253.84,1024.83,964.49$.

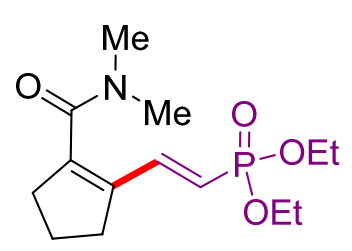

\section{Diethyl(E)-(2-(2-(dimethylcarbamoyl)cyclopent-1-en-1-yl)vinyl)phosph} onate (3ia).

Following the general experiment procedure, 3ia was obtained as a yellow liquid (76\%, $46 \mathrm{mg}) .{ }^{1} \mathrm{H}$ NMR (500 MHz, $\left.\mathrm{CDCl}_{3}\right): \delta=7.08$ (dd, $J=17.0$ $\mathrm{Hz}, J=21.5 \mathrm{~Hz}, 1 \mathrm{H}), 5.59$ (t, $J=18.5 \mathrm{~Hz}, 1 \mathrm{H}), 3.99-4.02(\mathrm{~m}, 4 \mathrm{H}), 2.97$ (s, 3H), 2.87 (s, 3H), 2.70 (t, $J$ $=7.0 \mathrm{~Hz}, 2 \mathrm{H}), 2.51(\mathrm{t}, J=7.5 \mathrm{~Hz}, 2 \mathrm{H}), 1.92-1.98(\mathrm{~m}, 2 \mathrm{H}), 1.25(\mathrm{t}, J=7.5 \mathrm{~Hz}, 6 \mathrm{H}) .{ }^{13} \mathrm{C} \mathrm{NMR}(125 \mathrm{MHz}$, $\left.\mathrm{CDCl}_{3}\right): \delta=168.75,143.64,141.35\left(\mathrm{~d}, J_{\mathrm{C}-\mathrm{P}}=6.3 \mathrm{~Hz}\right), 137.98\left(\mathrm{~d}, J_{\mathrm{C}-\mathrm{P}}=23.8 \mathrm{~Hz}\right), 117.11\left(\mathrm{~d}, J_{\mathrm{C}-\mathrm{P}}=\right.$ $190.0 \mathrm{~Hz}), 61.97,61.92,38.01,36.18,34.53,32.07,22.10,16.50,16.45$. HR-MS (ESI): $\mathrm{m} / \mathrm{z}$ calculated for $\mathrm{C}_{14} \mathrm{H}_{24} \mathrm{NO}_{4} \mathrm{P}:[\mathrm{M}+\mathrm{H}]^{+}: 302.1516$, found: 302.1521. FTIR $\left(\mathrm{KBr}, \mathrm{cm}^{-1}\right)$ : 3573.18, 3444.70, 3417.67, 2924.61, 1657.37, 1384.46, 1257.95, 1050.62, 1025.02, 964.09, 796.08.<smiles>CCCC(C)=C(C=CCP(=O)(OCC)OCC)C(=O)N(C)C</smiles>

\section{Diethyl((1E,3Z)-4-(dimethylcarbamoyl)deca-1,3-dien-1-yl)phosph}

onate (3ja). Following the general experiment procedure, $\mathbf{3 j a}$ was obtained as a yellow liquid $(67 \%, 46 \mathrm{mg}) .{ }^{1} \mathrm{H} \mathrm{NMR}\left(500 \mathrm{MHz}, \mathrm{CDCl}_{3}\right)$ : $\delta=6.88-6.98(\mathrm{~m}, 1 \mathrm{H}), 6.05-6.07(\mathrm{~m}, 1 \mathrm{H}), 5.72(\mathrm{dd}, J=17.0 \mathrm{~Hz}, J=19.0 \mathrm{~Hz}, 1 \mathrm{H}), 4.03-4.09(\mathrm{~m}, 4 \mathrm{H})$, $3.04(\mathrm{~s}, 3 \mathrm{H}), 2.95$ (s, 3H), $2.33(\mathrm{t}, J=7.0 \mathrm{~Hz}, 2 \mathrm{H}), 1.45-1.52(\mathrm{~m}, 2 \mathrm{H}), 1.28-1.33(\mathrm{~m}, 12 \mathrm{H}), 0.88(\mathrm{t}, J=$ $7.0 \mathrm{~Hz}, 3 \mathrm{H}) .{ }^{13} \mathrm{C}$ NMR $\left(125 \mathrm{MHz}, \mathrm{CDCl}_{3}\right): \delta=170.05,146.74,143.77\left(\mathrm{~d}, J_{\mathrm{C}-\mathrm{P}}=6.3 \mathrm{~Hz}\right), 125.56(\mathrm{~d}$, $\left.J_{\mathrm{C}-\mathrm{P}}=27.5 \mathrm{~Hz}\right), 118.71\left(\mathrm{~d}, J_{\mathrm{C}-\mathrm{P}}=190.0 \mathrm{~Hz}\right), 61.90,61.86,37.89,35.13,34.39,31.64,29.15,27.47$, 
22.61, 16.45, 16.40, 14.12. HR-MS (ESI): $\mathrm{m} / \mathrm{z}$ calculated for $\mathrm{C}_{17} \mathrm{H}_{32} \mathrm{NO}_{4} \mathrm{P}:[\mathrm{M}+\mathrm{H}]^{+}: 346.2142$, found: 346.2140. FTIR (KBr, $\left.\mathrm{cm}^{-1}\right): 3564.75,3444.56,3384.08,3362.78,2346.88,1611.53,1633.73$, 1384.18<smiles>CCOP(=O)(/C=C/C=C(/C)C(=O)N1CCCC1)OCC</smiles>
Diethyl((1E,3Z)-4-methyl-5-oxo-5-(pyrrolidin-1-yl)penta-1,3-dien-1-yl) phosphonate (3ka). Following the general experiment procedure, 3ka was obtained as a yellow liquid (78\%, $47 \mathrm{mg}) .{ }^{1} \mathrm{H} \mathrm{NMR}\left(500 \mathrm{MHz}, \mathrm{CDCl}_{3}\right): \delta$ $=6.92-7.02(\mathrm{~m}, 1 \mathrm{H}), 6.05(\mathrm{~d}, J=11.5 \mathrm{~Hz}, 1 \mathrm{H}), 5.71(\mathrm{dd}, J=17.0 \mathrm{~Hz}, J=18.5 \mathrm{~Hz}, 1 \mathrm{H}), 4.03-4.09(\mathrm{~m}$, 4H), $3.56(\mathrm{t}, J=7.0 \mathrm{~Hz}, 2 \mathrm{H}), 3.30(\mathrm{t}, J=6.0 \mathrm{~Hz}, 2 \mathrm{H}), 2.04(\mathrm{~s}, 3 \mathrm{H}), 1.90-1.96(\mathrm{~m}, 4 \mathrm{H}), 1.31(\mathrm{t}, J=7.0$ $\mathrm{Hz}, 6 \mathrm{H}) .{ }^{13} \mathrm{C} \mathrm{NMR}\left(125 \mathrm{MHz}, \mathrm{CDCl}_{3}\right): \delta=168.76,143.77\left(\mathrm{~d}, J_{\mathrm{C}-\mathrm{P}}=6.3 \mathrm{~Hz}\right), 143.19,126.69\left(\mathrm{~d}, J_{\mathrm{C}-\mathrm{P}}=\right.$ $27.5 \mathrm{~Hz}), 118.62\left(\mathrm{~d}, J_{\mathrm{C}-\mathrm{P}}=190.0 \mathrm{~Hz}\right), 61.97,61.93,47.57,45.39,26.04,24.49,20.72,16.50,16.44$. HR-MS (ESI): $\mathrm{m} / \mathrm{z}$ calculated for $\mathrm{C}_{14} \mathrm{H}_{24} \mathrm{NO}_{4} \mathrm{P}:[\mathrm{M}+\mathrm{H}]^{+}: 302.1516$, found: 302.1515 . FTIR (KBr, $\left.\mathrm{cm}^{-1}\right)$ : $3626.79,3606.16,3452.30,1633.74,1621.75,1615.23$.<smiles>CCOP(=O)(/C=C/C=C(/C)C(=O)N1CCCCC1)OCC</smiles>

\section{Diethyl((1E,3Z)-4-methyl-5-oxo-5-(piperidin-1-yl)penta-1,3-dien-1-yl)p} hosphonate (3la). Following the general experiment procedure, 3la was obtained as a yellow liquid $(51 \%, 32 \mathrm{mg}) .{ }^{1} \mathrm{H}$ NMR $\left(500 \mathrm{MHz}, \mathrm{CDCl}_{3}\right): \delta$ $=6.95-7.04(\mathrm{~m}, 1 \mathrm{H}), 6.06(\mathrm{~d}, J=11.5 \mathrm{~Hz}, 1 \mathrm{H}), 5.68(\mathrm{dd}, J=16.5 \mathrm{~Hz}, J=19.0 \mathrm{~Hz}, 1 \mathrm{H}), 4.03-4.09(\mathrm{~m}$, 4H), $3.64(\mathrm{t}, J=5.0 \mathrm{~Hz}, 2 \mathrm{H}), 3.34(\mathrm{t}, J=5.5 \mathrm{~Hz}, 2 \mathrm{H}), 2.04(\mathrm{~s}, 3 \mathrm{H}), 1.62-1.67(\mathrm{~m}, 4 \mathrm{H}), 1.53-1.54(\mathrm{~m}$, 2H), $1.31(\mathrm{t}, J=7.0 \mathrm{~Hz}, 6 \mathrm{H}) .{ }^{13} \mathrm{C} \mathrm{NMR}\left(125 \mathrm{MHz}, \mathrm{CDCl}_{3}\right): \delta=168.57,143.77\left(\mathrm{~d}, J_{\mathrm{C}-\mathrm{P}}=6.3 \mathrm{~Hz}\right)$, $142.40,126.62\left(\mathrm{~d}, J_{\mathrm{C}-\mathrm{P}}=27.5 \mathrm{~Hz}\right), 118.13\left(\mathrm{~d}, J_{\mathrm{C}-\mathrm{P}}=190.0 \mathrm{~Hz}\right), 61.91,61.87,47.52,42.25,26.66,25.67$, 24.58, 21.29, 16.48, 16.42. HR-MS (ESI): m/z calculated for $\mathrm{C}_{15} \mathrm{H}_{26} \mathrm{NO}_{4} \mathrm{P}:[\mathrm{M}+\mathrm{H}]^{+}:$316.1672, found: 316.1670. FTIR $\left(\mathrm{KBr}, \mathrm{cm}^{-1}\right): 3646.13,3507.40,3384.04,2455.33,1651.53,1621.58,1614.85$, 1384.22<smiles>CCOP(=O)(/C=C/C=C(/C)C(=O)N(CC)CC)OCC</smiles>

\section{Diethyl((1E,3Z)-5-(diethylamino)-4-methyl-5-oxopenta-1,3-dien-1-yl)p} hosphonate (3ma). Following the general experiment procedure, $3 \mathrm{ma}$ was obtained as a yellow liquid $(76 \%, 46 \mathrm{mg}) .{ }^{1} \mathrm{H}$ NMR $\left(500 \mathrm{MHz}, \mathrm{CDCl}_{3}\right): \delta$ $=6.90-7.00(\mathrm{~m}, 1 \mathrm{H}), 6.05(\mathrm{~d}, J=11.0,1 \mathrm{H}), 5.69(\mathrm{dd}, J=16.5, J=19.5$, 
1H), 4.02-4.08 (m, 4H), 3.47-3.49 (m, 2H), 3.28 (q, $J=7.5,2 \mathrm{H}), 2.05(\mathrm{~s}, 3 \mathrm{H}), 1.31(\mathrm{t}, J=7.0,6 \mathrm{H})$, $1.22(\mathrm{t}, J=7.0,3 \mathrm{H}), 1.15(\mathrm{t}, J=7.5,3 \mathrm{H}) .{ }^{13} \mathrm{C} \mathrm{NMR}\left(125 \mathrm{MHz}, \mathrm{CDCl}_{3}\right): \delta=169.69,143.65\left(\mathrm{~d}, J_{\mathrm{C}-\mathrm{P}}=\right.$ $5.0 \mathrm{~Hz}), 142.71,126.01\left(\mathrm{~d}, J_{\mathrm{C}-\mathrm{P}}=27.5 \mathrm{~Hz}\right), 118.11\left(\mathrm{~d}, J_{\mathrm{C}-\mathrm{P}}=152.5 \mathrm{~Hz}\right), 61.93,61.88,42.60,38.60$, 21.33, 16.48, 16.42, 14.38, 12.94. HR-MS (ESI): $\mathrm{m} / \mathrm{z}$ calculated for $\mathrm{C}_{14} \mathrm{H}_{26} \mathrm{NO}_{4} \mathrm{P}:[\mathrm{M}+\mathrm{H}]^{+}: 304.1672$, found: 304.1671 . FTIR $\left(\mathrm{KBr}, \mathrm{cm}^{-1}\right): 3850.90,3646.05,3444.62,1651.48,1557.23,1384.12$.

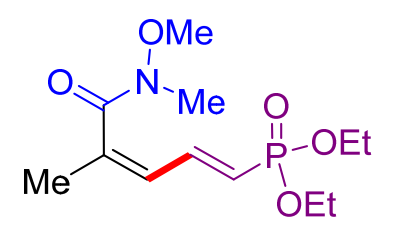

Diethyl((1E,3Z)-5-(methoxy(methyl)amino)-4-methyl-5-oxopenta-1,3-d ien-1-yl)phosphonate (3na).

Following the general experiment procedure, 3na was obtained as a yellow liquid $(69 \%, 40 \mathrm{mg}) .{ }^{1} \mathrm{H}$ NMR $\left(500 \mathrm{MHz}, \mathrm{CDCl}_{3}\right): \delta=6.94-7.04(\mathrm{~m}, 1 \mathrm{H}), 6.04(\mathrm{~d}, J=11.0$ Hz, 1H), $5.65(\mathrm{t}, J=18.0 \mathrm{~Hz}, 1 \mathrm{H}), 3.98-4.01(\mathrm{~m}, 4 \mathrm{H}), 3.52(\mathrm{~s}, 3 \mathrm{H}), 3.19(\mathrm{~s}, 3 \mathrm{H}), 1.99$ (s, 3H), $1.24(\mathrm{t}, J$ $=7.0 \mathrm{~Hz}, 6 \mathrm{H}) \cdot{ }^{13} \mathrm{C}$ NMR $\left(125 \mathrm{MHz}, \mathrm{CDCl}_{3}\right): \delta=169.69,143.65\left(\mathrm{~d}, J_{\mathrm{C}-\mathrm{P}}=6.3 \mathrm{~Hz}\right), 142.70,126.49(\mathrm{~d}$, $\left.J_{\mathrm{C}-\mathrm{P}}=26.3 \mathrm{~Hz}\right), 118.04\left(\mathrm{~d}, J_{\mathrm{C}-\mathrm{P}}=190.0 \mathrm{~Hz}\right), 61.91\left(\mathrm{~d}, J_{\mathrm{C}-\mathrm{P}}=6.3 \mathrm{~Hz}\right), 42.59,38.59,21.28,16.42\left(\mathrm{~d}, J_{\mathrm{C}-\mathrm{P}}\right.$ $=6.3 \mathrm{~Hz}), 14.34,12.90$. HR-MS (ESI): $\mathrm{m} / \mathrm{z}$ calculated for $\mathrm{C}_{12} \mathrm{H}_{22} \mathrm{NO}_{5} \mathrm{P}:[\mathrm{M}+\mathrm{H}]^{+}: 292.1308$, found: 292.1307. FTIR $\left(\mathrm{KBr}, \mathrm{cm}^{-1}\right): 3673.52,3444.63,2345.33,1667.35,1644.66,1614.98,1384.39$, 1019.70.<smiles>CCOP(=O)(/C=C/C=C(/C)C(=O)NC(C)C)OCC</smiles>

\section{Diethyl((1E,3Z)-4-methyl-5-(methylamino)-5-oxopenta-1,3-dien-1-yl)p}

hosphonate (3oa). Following the general experiment procedure, 3oa was obtained as a yellow liquid (64\%, $33 \mathrm{mg}) .{ }^{1} \mathrm{H}$ NMR $\left(500 \mathrm{MHz}, \mathrm{CDCl}_{3}\right): \delta=7.26-7.36(\mathrm{~m}, 1 \mathrm{H}), 6.08(\mathrm{~d}$, $J=11.5 \mathrm{~Hz}, 1 \mathrm{H}), 6.06$ (brs, 1H), $5.65(\mathrm{dd}, J=16.5 \mathrm{~Hz}, J=19.0 \mathrm{~Hz}, 1 \mathrm{H}), 3.98-4.03$ (m, 4H), 2.84 (d, $J$ $=4.5 \mathrm{~Hz} 3 \mathrm{H}), 1.98(\mathrm{~s}, 3 \mathrm{H}), 1.25(\mathrm{t}, J=7.0 \mathrm{~Hz}, 6 \mathrm{H}) .{ }^{13} \mathrm{C} \mathrm{NMR}\left(125 \mathrm{MHz}, \mathrm{CDCl}_{3}\right): \delta=169.26,143.72$ $\left(\mathrm{d}, J_{\mathrm{C}-\mathrm{P}}=7.5 \mathrm{~Hz}\right), 141.58,129.64\left(\mathrm{~d}, J_{\mathrm{C}-\mathrm{P}}=27.5 \mathrm{~Hz}\right), 119.47\left(\mathrm{~d}, J_{\mathrm{C}-\mathrm{P}}=188.8 \mathrm{~Hz}\right), 62.07,62.02,26.53$, 21.49, 16.50, 16.45. HR-MS (ESI): $\mathrm{m} / \mathrm{z}$ calculated for $\mathrm{C}_{11} \mathrm{H}_{20} \mathrm{NO}_{4} \mathrm{P}:[\mathrm{M}+\mathrm{H}]^{+}: 262.1203$, found: 262.1206. FTIR (KBr, cm $\left.{ }^{-1}\right): 3473.10,3423.80,1651.54,1644.83,1615.11,1384.35,1024.30$. 
<smiles>CC(=CC=CS(=O)(=O)c1ccccc1)C(=O)N(C)C</smiles>

(2Z,4E)- $N, N, 2-$ trimethyl-5-(phenylsulfonyl)penta-2,4-dienamide (3ab). 7.59-7.63 (m, 1H), $7.53(\mathrm{~m}, 2 \mathrm{H}), 7.10(\mathrm{dd}, J=11.5 \mathrm{~Hz}, J=14.5 \mathrm{~Hz}, 1 \mathrm{H}), 6.36(\mathrm{~d}, J=14.5 \mathrm{~Hz}, 1 \mathrm{H})$, $6.04(\mathrm{~d}, J=11.5 \mathrm{~Hz}, 1 \mathrm{H}), 3.07(\mathrm{~s}, 3 \mathrm{H}), 2.96(\mathrm{~s}, 3 \mathrm{H}), 2.06(\mathrm{~s}, 3 \mathrm{H}) .{ }^{13} \mathrm{C} \mathrm{NMR}\left(125 \mathrm{MHz}, \mathrm{CDCl}_{3}\right): \delta=$ 169.64, 145.97, 140.44, 137.48, 133.51, 130.94, 129.38, 127.72, 123.36, 37.76, 34.44, 21.26. HR-MS (ESI): $\mathrm{m} / \mathrm{z}$ calculated for $\mathrm{C}_{14} \mathrm{H}_{17} \mathrm{NO}_{3} \mathrm{~S}:[\mathrm{M}+\mathrm{H}]^{+}:$280.1002, found: 280.1009. FTIR $\left(\mathrm{KBr}, \mathrm{cm}^{-1}\right)$ : $3507.57,3473.04,3444.51,3383.77,1633.72,1621.71,1384.25$.<smiles>CN(C)C(=O)/C(=C\C=C\S(=O)(=O)c1ccccc1)c1ccccc1</smiles>

$(2 Z, 4 E)-N, N$-dimethyl-2-phenyl-5-(phenylsulfonyl)penta-2,4-diena mide (3bb). Following the general experiment procedure, $3 \mathbf{b b}$ was obtained as a yellow liquid $(82 \%, 56 \mathrm{mg}) .{ }^{1} \mathrm{H}$ NMR $\left(500 \mathrm{MHz}, \mathrm{CDCl}_{3}\right)$ : $\delta=7.89-7.91(\mathrm{~m}, 2 \mathrm{H}), 7.62(\mathrm{t}, J=7.5 \mathrm{~Hz}, 1 \mathrm{H}), 7.54(\mathrm{t}, J=7.5 \mathrm{~Hz}, 2 \mathrm{H}), 7.34-7.44(\mathrm{~m}, 5 \mathrm{H}), 7.31(\mathrm{dd}, J$ $=11.5 \mathrm{~Hz}, J=14.5 \mathrm{~Hz}, 1 \mathrm{H}), 6.65(\mathrm{~d}, J=11.5 \mathrm{~Hz}, 1 \mathrm{H}), 6.55(\mathrm{~d}, J=14.5 \mathrm{~Hz}, 1 \mathrm{H}), 3.18(\mathrm{~s}, 3 \mathrm{H}), 2.88(\mathrm{~s}$, $3 \mathrm{H}) .{ }^{13} \mathrm{C}$ NMR $\left(125 \mathrm{MHz}, \mathrm{CDCl}_{3}\right): \delta=168.04,147.69,140.36,137.64,134.14,133.59,132.50,130.12$, 129.44, 129.28, 127.83, 126.29, 121.34, 38.04, 34.68. HR-MS (ESI): m/z calculated for $\mathrm{C}_{19} \mathrm{H}_{19} \mathrm{NO}_{3} \mathrm{~S}$ : $[\mathrm{M}+\mathrm{H}]^{+}:$342.1158, found: 342.1154. FTIR $\left(\mathrm{KBr}, \mathrm{cm}^{-1}\right): 3616.80,3444.91,2924.95,1651.07,1633.70$, $1504.65,1445.99,1306.00,1144.69,1084.41,829.00,753.37,688.22,591.24$.<smiles>CC/C=C(C)/C(=C\C=C\S(=O)(=O)c1ccccc1)C(=O)N(C)C</smiles>

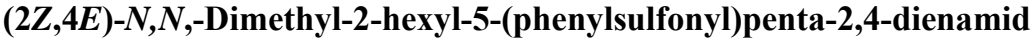
e (3jb).

Following the general experiment procedure, $\mathbf{3 j b}$ was obtained as a yellow liquid $(93 \%, 65 \mathrm{mg}) .{ }^{1} \mathrm{H}$ NMR $\left(500 \mathrm{MHz}, \mathrm{CDCl}_{3}\right): \delta=7.86-7.87$ $(\mathrm{m}, 2 \mathrm{H}), 7.60-7.63(\mathrm{~m}, 1 \mathrm{H}), 7.51-7.55(\mathrm{~m}, 2 \mathrm{H}), 7.11(\mathrm{dd}, J=11.5 \mathrm{~Hz}, J=15.0 \mathrm{~Hz}, 1 \mathrm{H}), 6.37(\mathrm{~d}, J=$ $14.5 \mathrm{~Hz}, 1 \mathrm{H}), 6.03$ (d, $J=11.5 \mathrm{~Hz}, 1 \mathrm{H}), 3.08(\mathrm{~s}, 3 \mathrm{H}), 2.95(\mathrm{~s}, 3 \mathrm{H}), 2.35$ (t, $J=7.5 \mathrm{~Hz}, 2 \mathrm{H}), 1.44-1.50$ (m, 2H), 1.26-1.33 (m, 6H), $0.87(\mathrm{t}, J=6.0 \mathrm{~Hz}, 3 \mathrm{H}) .{ }^{13} \mathrm{C} \mathrm{NMR}\left(125 \mathrm{MHz}, \mathrm{CDCl}_{3}\right): \delta=169.47,150.55$, $140.45,137.62,133.50,131.00,129.38,127.74,122.02,37.90,35.34,34.48,31.57,29.08,27.36,22.56$, 14.09. HR-MS (ESI): $\mathrm{m} / \mathrm{z}$ calculated for $\mathrm{C}_{19} \mathrm{H}_{27} \mathrm{NO}_{3} \mathrm{~S}$ : $[\mathrm{M}+\mathrm{H}]^{+}: 350.1784$, found: 350.1783 . FTIR (KBr, 
$\left.\mathrm{cm}^{-1}\right): 3851.02,3444.53,3299.54,3262.31,2924.11,1615.03,1384.26,1144.60,1084.43,830.25$, 752.73.

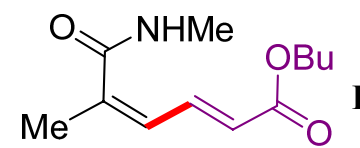

Butyl(2E,4Z)-5-methyl-6-(methylamino)-6-oxohexa-2,4-dienoate (3oc).

Following the general experiment procedure, 3oc was obtained as a yellow liquid $(71 \%, 32 \mathrm{mg}) .{ }^{1} \mathrm{H}$ NMR (500 MHz, $\left.\mathrm{CDCl}_{3}\right): \delta=7.55(\mathrm{dd}, J=11.5 \mathrm{~Hz}, J=15.0 \mathrm{~Hz}, 1 \mathrm{H}), 6.95(\mathrm{~d}, J=11.5 \mathrm{~Hz}, 1 \mathrm{H}), 6.10$ $(\mathrm{d}, J=15.5 \mathrm{~Hz}, 1 \mathrm{H}), 5.95(\mathrm{~s}, 1 \mathrm{H}), 4.18(\mathrm{t}, J=6.5 \mathrm{~Hz}, 2 \mathrm{H}), 2.91(\mathrm{~d}, J=5.0 \mathrm{~Hz}, 3 \mathrm{H}), 2.08(\mathrm{~s}, 3 \mathrm{H})$, 1.64-1.69 (m, 2H), 1,37-1.43 (m, 2H), $0.95(\mathrm{t}, J=7.5 \mathrm{~Hz}, 3 \mathrm{H}) .{ }^{13} \mathrm{C}$ NMR $\left(125 \mathrm{MHz}, \mathrm{CDCl}_{3}\right): \delta=$ $167.76,165.68,137.45,137.15,129.16,124.84,63.60,29.67,25.76,18.14,12.71,12.63 .{ }^{13} \mathrm{C}$ NMR $\left(125 \mathrm{MHz}, \mathrm{CDCl}_{3}\right): \delta=168.90,166.82,138.59,138.29,130.30,125.98,64.74,30.82,26.90,19.28$, $13.85,13.77$. 


\section{Deuterium-Labeled Experiments}

\section{H/D Exchange in 1b}

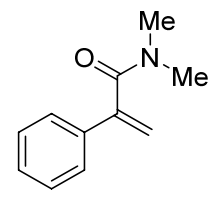

$1 \mathrm{~b}$

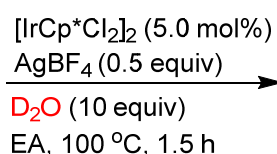

EA, $100^{\circ} \mathrm{C}, 1.5 \mathrm{~h}$

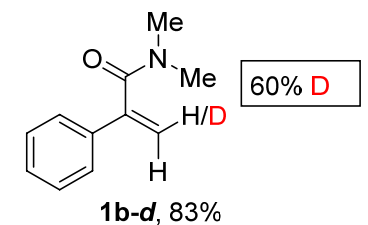

$1 b-d, 83 \%$

An oven-dried vial was charged with $\left[\mathrm{IrCp}^{*} \mathrm{Cl}_{2}\right]_{2}(10 \mathrm{~mol} \%, 0.02 \mathrm{mmol}), \operatorname{AgBF}_{4}(0.5$ equiv, 0.1 mmol), EtOAc $(1 \mathrm{~mL})$ and $\mathrm{D}_{2} \mathrm{O}(10$ equiv, $2.0 \mathrm{mmol})$. Then, acrylamide $\mathbf{1 b}(35.0 \mathrm{mg}, 0.2 \mathrm{mmol})$ was added into the solution. The vial was sealed under argon and heated to $100^{\circ} \mathrm{C}$ with stirring for 1.5 hours. After cooling down, the mixture was directly applied to a flash column chromatography $\left(\mathrm{CH}_{3} \mathrm{OH} / \mathrm{CH}_{2} \mathrm{Cl}_{2}\right.$ mixtures) on silica gel to afford $\mathbf{1 b}-\mathbf{d}(29.0 \mathrm{mg}, 83 \%$ recovered). The D\% of $\mathbf{1 b}-\boldsymbol{d}$ was estimated by ${ }^{1} \mathrm{H}$ NMR.

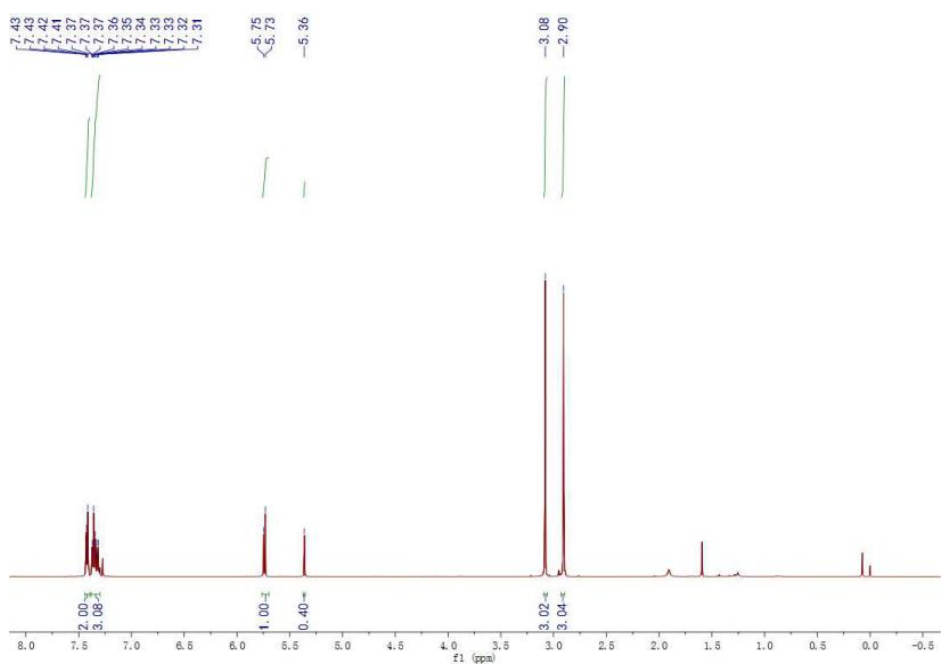




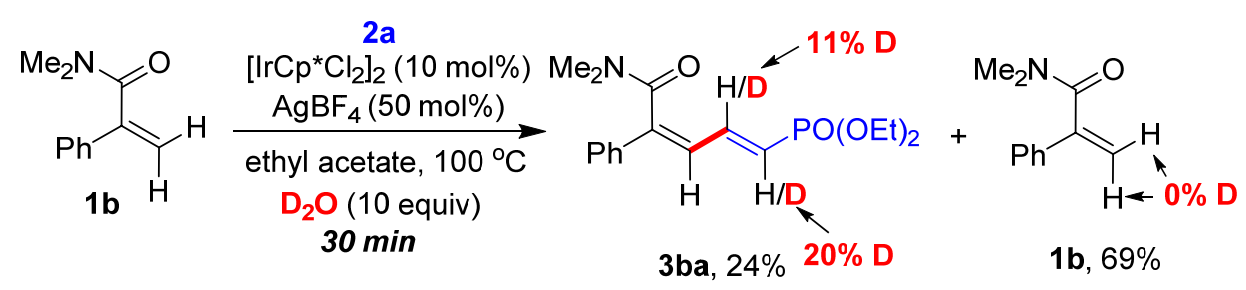

An oven-dried vial was charged with $\left[\mathrm{IrCp}^{*} \mathrm{Cl}_{2}\right]_{2}(10 \mathrm{~mol} \%, 0.02 \mathrm{mmol}), \operatorname{AgBF}_{4}(0.5$ equiv, 0.1 mmol), EtOAc $(1 \mathrm{~mL})$ and $\mathrm{D}_{2} \mathrm{O}(10$ equiv, $2.0 \mathrm{mmol})$. Then, acrylamide $\mathbf{1 b}(35.0 \mathrm{mg}, 0.2 \mathrm{mmol})$ and vinyl phosphate $\mathbf{2 a}(0.4 \mathrm{mmol})$ were added into the solution. The vial was sealed under argon and heated to $100^{\circ} \mathrm{C}$ with stirring for 0.5 hour. After cooling down, the mixture was directly applied to a flash column chromatography $\left(\mathrm{CH}_{3} \mathrm{OH} / \mathrm{CH}_{2} \mathrm{Cl}_{2}\right.$ mixtures) on silica gel to afford $3 \mathbf{b a}$ (16.4 $\mathrm{mg}, 24 \%$ yield) and unreacted $\mathbf{1 b}\left(24.1 \mathrm{mg}, 69 \%\right.$ recovered). The D\% of $\mathbf{3 b a}$ and $\mathbf{1 b}$ were estimated by ${ }^{1} \mathrm{H}$ NMR.

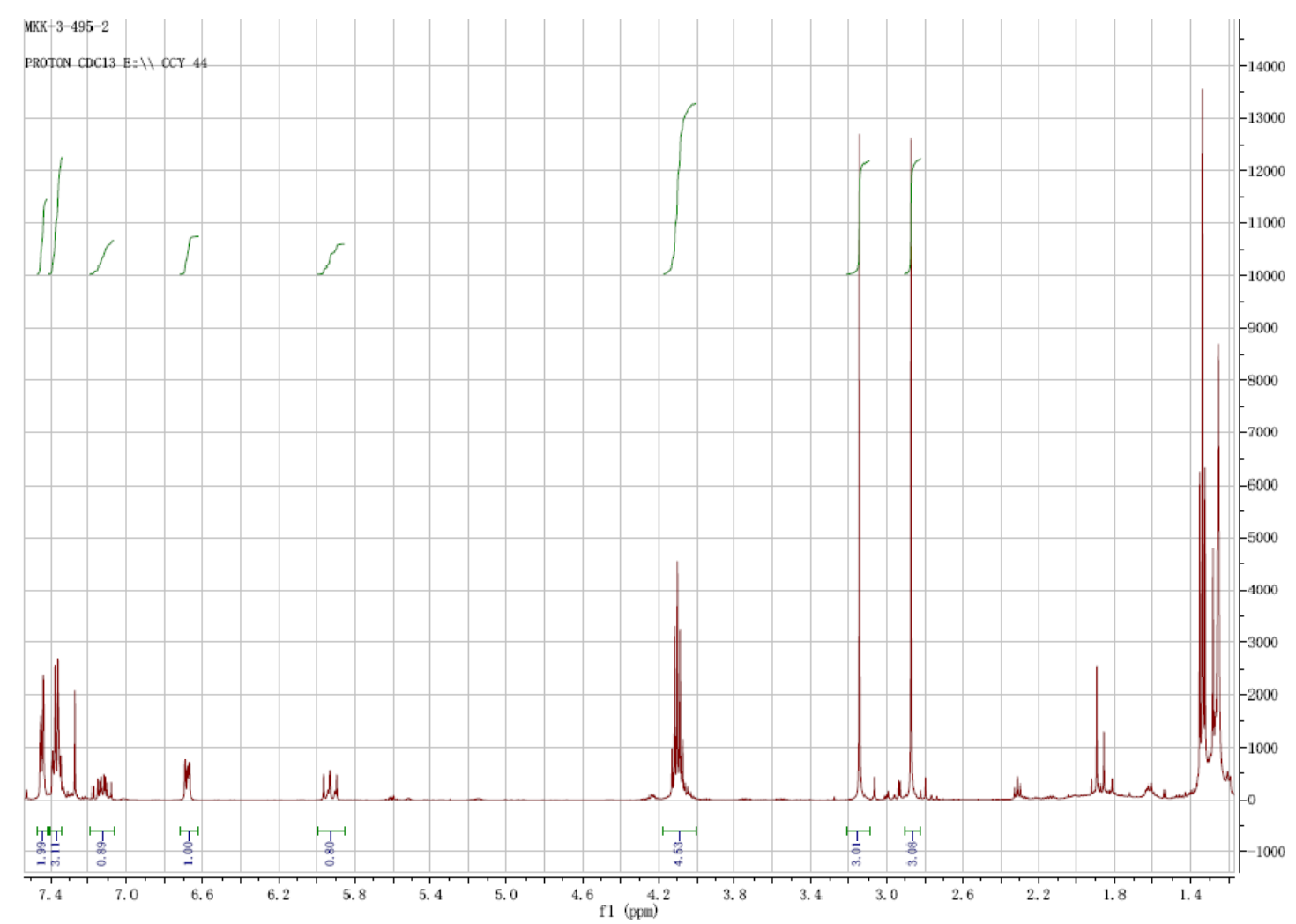




\section{Crossover Experiment}

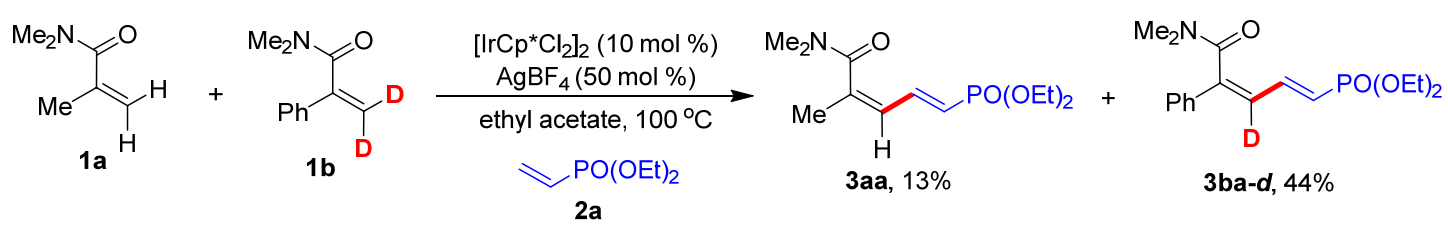

A $10 \mathrm{~mL}$ vial was charged with $\left[\mathrm{IrCp}^{*} \mathrm{Cl}_{2}\right]_{2}(15.9 \mathrm{mg}, 10 \mathrm{~mol} \%), \mathrm{AgBF}_{4}(19.5 \mathrm{mg}, 50 \mathrm{~mol} \%)$ and EtOAc $(1.0 \mathrm{~mL})$. Then, alkene $(32.83 \mathrm{mg}, 0.2 \mathrm{mmol}), N, N$-dimethyl methacrylamide 1a $(11.3 \mathrm{mg}, 0.1$ mmol) and $N, N$-dimethyl-2-phenylacrylamide- $d_{2} \mathbf{1 b}(17.7 \mathrm{mg}, 0.1 \mathrm{mmol})$ were added into the solution in sequence. The vial was sealed under $\mathrm{Ar}$ and heated to $100^{\circ} \mathrm{C}$ with stirring for 3 hours. After cooling down, the mixture was directly applied to column chromatography for separation to afford products 3aa ( $8.8 \mathrm{mg}, 13 \%$ yield) and 3ba-d (24.3 mg, 44\% yield). 


\section{Competitive KIE Experiment with $1 \mathrm{~b}$ and $1 \mathrm{~b}-\mathrm{d}_{2}$}

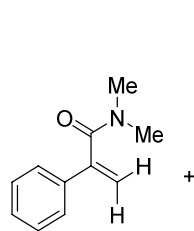

$1 b$

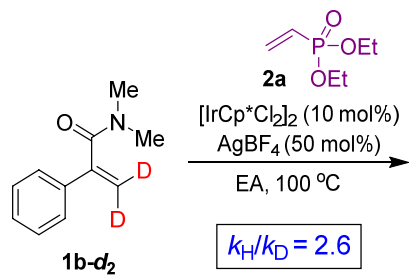

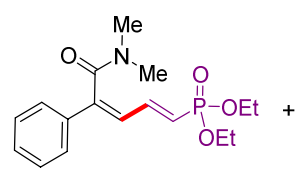

3 ba

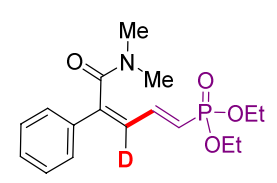

3ba-d

A $10 \mathrm{~mL}$ vial was charged with $\left[\operatorname{IrCp}^{*} \mathrm{Cl}_{2}\right]_{2}(7.9 \mathrm{mg}, 10 \mathrm{~mol} \%), \mathrm{AgBF}_{4}(9.8 \mathrm{mg}, 50 \mathrm{~mol} \%)$ and EtOAc (1.0 mL). Then, alkene $5 \mathbf{a}(0.04 \mathrm{mmol})$, acrylamide $\mathbf{4 b}(17.5 \mathrm{mg}, 0.1 \mathrm{mmol})$ and $\mathbf{4 b}-\boldsymbol{d}_{2}(17.7 \mathrm{mg}$, $0.1 \mathrm{mmol}$ ) were added into the solution in sequence. The vial was sealed under $\mathrm{Ar}$ and heated to $100^{\circ} \mathrm{C}$ with stirring for 6 hours. After cooling down, the mixture was directly applied to column chromatography for separation. The ratio of $\mathbf{6 b a} / \mathbf{6 b a}-\mathbf{d}_{\mathbf{2}}\left(13.0 \mathrm{mg}, 19 \%\right.$ yield) was determined by ${ }^{1} \mathrm{H}$ NMR to be 2.6.

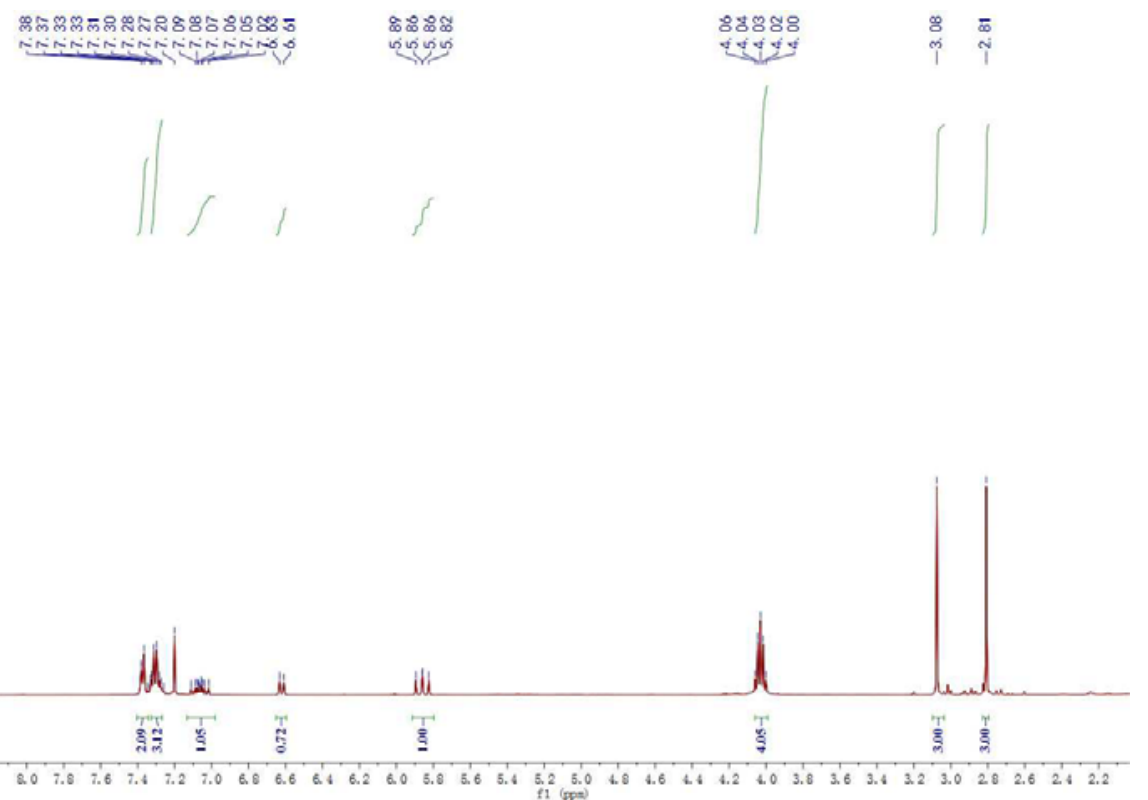




\section{Parallel KIE Experiments with $1 \mathrm{~b}$ or $1 \mathrm{~b}-\boldsymbol{d}_{2}$}

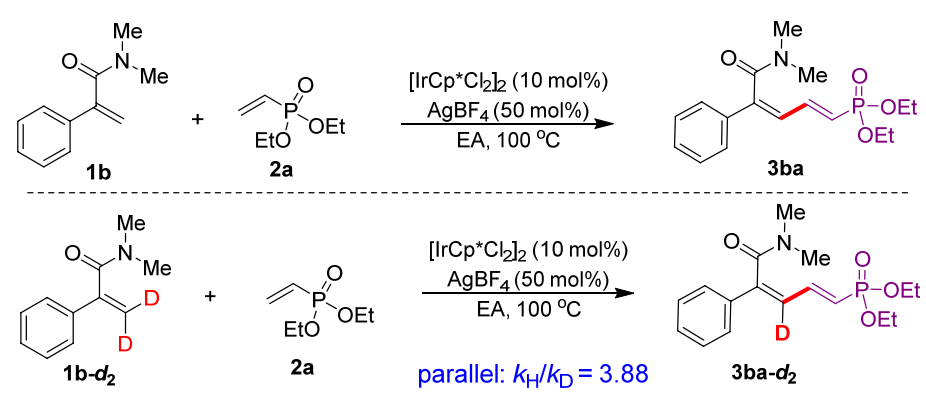

Parallel independent reactions of $\mathbf{1 b}$ or $\mathbf{1 b}-\boldsymbol{d}_{\mathbf{2}}$ with $\mathbf{2 a}$ were performed to determine the corresponding KIE value. Each $10 \mathrm{~mL}$ vial was charged with $\left[\operatorname{IrCp}^{*} \mathrm{Cl}_{2}\right]_{2}(7.9 \mathrm{mg}, 10 \mathrm{~mol} \%), \mathrm{AgBF}_{4}$ (9.8 mg, $50 \mathrm{~mol} \%)$, EtOAc (1.5 mL). Then, vinylphosphonate 2a (32.8 mg, 0.2mmol, 2.0 equiv), acrylamide $\mathbf{1 b}$ (17.5 mg, $0.1 \mathrm{mmol}, 1.0$ equiv) or $\mathbf{1 b}-\mathrm{d}_{2}(17.7 \mathrm{mg}, 0.1 \mathrm{mmol}, 1.0$ equiv) were added into the solution in sequence. The vials were sealed under $\mathrm{Ar}$ and heated to $100^{\circ} \mathrm{C}$ with stirring for $7,14,21$ or 28 minutes. After cooling down, the mixture was concentrated in vacuo and purified by column chromatography to determine the product yields. A KIE value was determined to be 3.88 .

\begin{tabular}{c|c|c|c|c}
\hline yield & 7 & 14 & 21 & 28 \\
\hline 6ba & $15.7 \%$ & $18.9 \%$ & $24.1 \%$ & $27.7 \%$ \\
\hline 6ba-d $_{2}$ & $3.1 \%$ & $3.7 \%$ & $4.7 \%$ & $6.3 \%$ \\
\hline
\end{tabular}

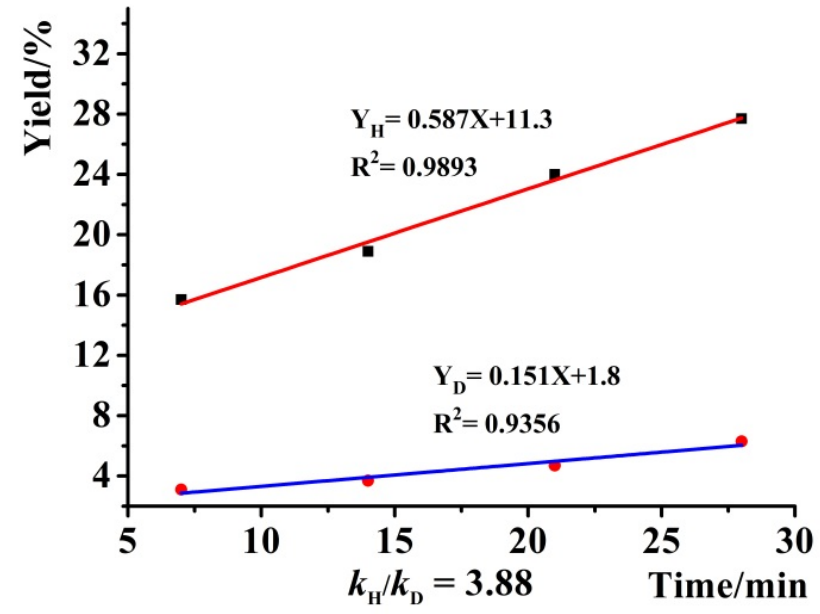




\section{Competitive Reaction of Different Acrylamides 1e and $1 f$}

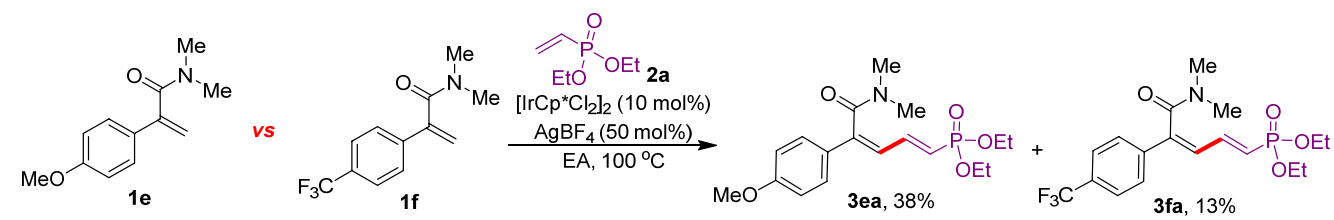

A $10 \mathrm{~mL}$ vial was charged with $\left[\mathrm{IrCp}^{*} \mathrm{Cl}_{2}\right]_{2}(15.9 \mathrm{mg}, 10 \mathrm{~mol} \%), \mathrm{AgBF}_{4}(19.5 \mathrm{mg}, 0.5$ equiv) and EtOAc $(1 \mathrm{~mL})$. Then, alkene 2a (32.9 mg, $0.2 \mathrm{mmol})$, acrylamide $1 \mathbf{e}(41.1 \mathrm{mg}, 0.2 \mathrm{mmol})$ and $\mathbf{1 f}(48.7$ $\mathrm{mg}, 0.2 \mathrm{mmol}$ ) were added into the solution in sequence. The vial was sealed under Ar and heated to $100^{\circ} \mathrm{C}$ with stirring for 3 hours. After cooling down, the mixture was concentrated in vacuo and purified by column chromatography to afford the product 3ea $(27.8 \mathrm{mg}, 38 \%)$ and $\mathbf{3 f a}(10.2 \mathrm{mg}, 13 \%)$. 


\section{Hammett-Plot with Analysis}

Independent reactions of $\mathbf{2 a}$ with 1a-1e were performed to determine the initial rates for differently substrates. Acrylamides $\mathbf{1 b}(17.5 \mathrm{mg}, 0.1 \mathrm{mmol})$ or $1 \mathbf{e}(20.5 \mathrm{mg}, 0.1 \mathrm{mmol})$ or $1 \mathbf{d}(19.3 \mathrm{mg}, 0.1 \mathrm{mmol})$ or 1 c $(21 \mathrm{mg}, 0.1 \mathrm{mmol})$ or $\mathbf{1 f}(24.3 \mathrm{mg}, 0.1 \mathrm{mmol}),\left[\mathrm{IrCp}^{*} \mathrm{Cl}_{2}\right]_{2}(7.9 \mathrm{mg}, 10 \mathrm{~mol} \%), \mathrm{AgBF}_{4}(9.8 \mathrm{mg}, 50$ mol \%) and EtOAc $(1.0 \mathrm{~mL})$ were placed in a $10 \mathrm{~mL}$ vial. The vials were sealed under $\mathrm{Ar}$ and heated to $100^{\circ} \mathrm{C}$ with stirring for $2,4,6,8$ or 10 minutes. After cooling down, the mixture was concentrated in vacuo and the yield was calculated by NMR using 1,3,5-trimethoxybenzene as the interior label.

\begin{tabular}{|l|l|l|l|l|l|l|l|l|}
\hline $\mathrm{t} / \mathrm{min}$ & 2 & 4 & 6 & 8 & 10 & $\boldsymbol{\sigma}_{\mathbf{p}}$ & $\boldsymbol{k} / \mathbf{1 0} \mathbf{0}^{-4} \mathbf{m m o l} / \mathbf{m i n}$ & $\mathbf{l o g}\left(\boldsymbol{k}_{X} / \boldsymbol{k}_{H}\right)$ \\
\hline 3ba yield (\%) & 10.0 & 12.5 & 14.4 & 15 & 16.5 & 0.00 & 0.845 & 0.00 \\
\hline 3ea yield (\%) & 11.4 & 14.5 & 17.2 & 19.8 & 20.9 & -0.27 & 1.325 & 0.195 \\
\hline 3da yield (\%) & 6.1 & 7.2 & 8.9 & 10.0 & 11.4 & 0.14 & 0.670 & -0.100 \\
\hline 3ca yield (\%) & 5.3 & 6.8 & 7.5 & 8.9 & 9.7 & 0.23 & 0.545 & -0.190 \\
\hline 3fa yield (\%) & 3.4 & 4.2 & 5.5 & 6.4 & 6.9 & 0.54 & 0.460 & -0.260 \\
\hline
\end{tabular}

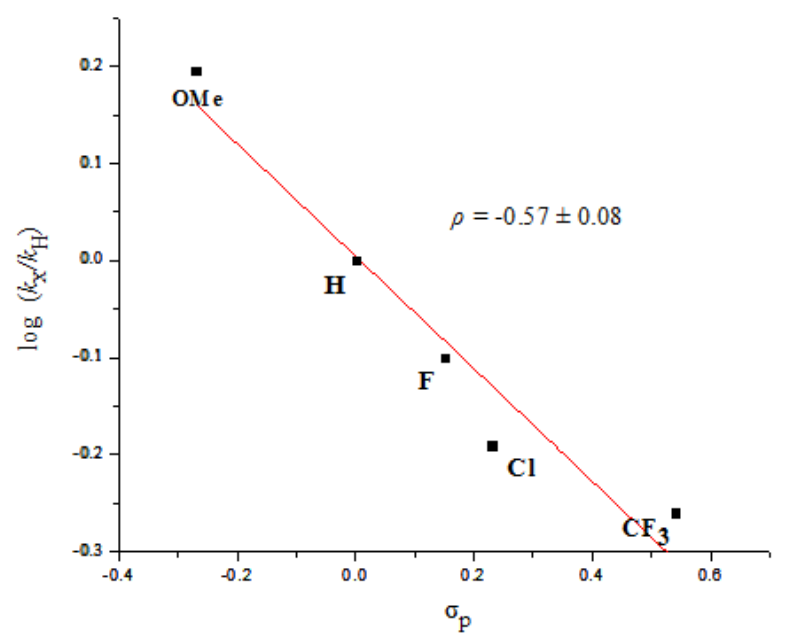




\section{Gram-Scale Synthesis of 3aa}

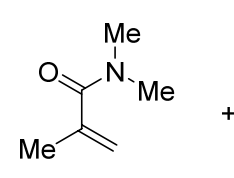

$1 \mathrm{a}, 0.90 \mathrm{~g}$

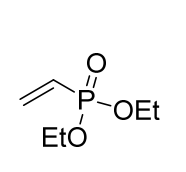

$2 \mathbf{a}$

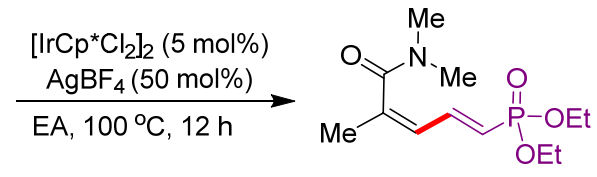

3aa, $61 \%$

An oven-dried vial was charged with $\left[\operatorname{IrCp}^{*} \mathrm{Cl}_{2}\right]_{2}(318.0 \mathrm{mg}, 5 \mathrm{~mol} \%), \mathrm{AgBF}_{4}(780.0 \mathrm{mg}, 50 \mathrm{~mol} \%)$ and EtOAc $(20 \mathrm{~mL})$. Then, alkene 1a (16.0 mmol, 2.0 equiv) and acrylamide 2a ( $0.90 \mathrm{~g}, 8.0 \mathrm{mmol}, 1.0$ equiv) were added into the solution in sequence. The vial was sealed under argon and heated to $100{ }^{\circ} \mathrm{C}$ with stirring for 12 hours. After cooling down, the mixture was concentrated to give the crude product which was directly applied to a flash column chromatography for purification $\left(\mathrm{CH}_{3} \mathrm{OH} / \mathrm{CH}_{2} \mathrm{Cl}_{2}\right.$ mixtures). The desired product 3aa was obtained as yellow oil (1.33 g, 61\%).

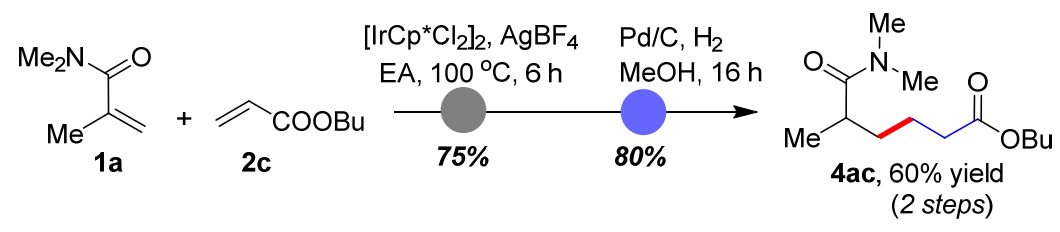

An oven-dried vial was charged with $\left[\mathrm{IrCp}^{*} \mathrm{Cl}_{2}\right]_{2}(131.8 \mathrm{mg}, 10 \mathrm{~mol} \%), \mathrm{AgBF}_{4}(39.0 \mathrm{mg}, 50 \mathrm{~mol} \%)$ and EtOAc $(2 \mathrm{~mL})$. Then, acrylate $\mathbf{2 c}(0.8 \mathrm{mmol}, 2.0$ equiv) and acrylamide 1a (45.2 mg, $0.4 \mathrm{mmol}, 1.0$ equiv) were added into the solution in sequence. The vial was sealed under argon and heated to $100{ }^{\circ} \mathrm{C}$ with stirring for 6 hours. After cooling down, the mixture was concentrated to give the crude product which was directly applied to a flash column chromatography for purification $\left(\mathrm{MeOH} / \mathrm{CH}_{2} \mathrm{Cl}_{2}\right.$ mixtures) to afford dienoate 3ac as a light yellow oil (72.4 mg, 75\% yield). Next, the obtained dienoate 3ac was dissolved in anhydrous methanol $(2 \mathrm{~mL})$, and $5 \% \mathrm{Pd} / \mathrm{C}$ powder $(30 \mathrm{wt} \%, 21 \mathrm{mg})$ was added into the solution. The mixture was then sealed and a balloon of hydrogen was introduced. The reaction was stirred at room temperature for overnight and the reaction was complete as indicated by TLC. The reaction mixture was filtered over celite and the filtrate was concentrated in vacuum. The crude product was purified by column chromatography on silica gel using 3:1 PE/EA as eluent to afford the product 4ac (58 mg, 80\% yield) as a yellow oil. ${ }^{1} \mathrm{H}$ NMR (500 MHz, $\left.\mathrm{CDCl}_{3}\right): \delta=4.06(\mathrm{t}, J=6.5 \mathrm{~Hz}, 2 \mathrm{H}), 3.05$, (s, 3H), $2.95(\mathrm{~s}, 3 \mathrm{H}), 2.70-2.74(\mathrm{~m}, 1 \mathrm{H}), 2.27-2.31(\mathrm{~m}, 2 \mathrm{H}), 1.68-1.75(\mathrm{~m}, 1 \mathrm{H}), 1.57-1.63(\mathrm{~m}, 4 \mathrm{H})$, $1.35-1.43(\mathrm{~m}, 3 \mathrm{H}), 1.10(\mathrm{~d}, J=7.0 \mathrm{~Hz}, 3 \mathrm{H}), 0.93(\mathrm{t}, J=7.5 \mathrm{~Hz}, 3 \mathrm{H}) .{ }^{13} \mathrm{C} \mathrm{NMR}\left(125 \mathrm{MHz}, \mathrm{CDCl}_{3}\right): \delta=$ 
$176.18,173.60,64.16,37.16,35.60,35.42,34.24,33.44,30.65,22.93,19.11,17.39,13.68$. HR-MS

(ESI): $\mathrm{m} / \mathrm{z}$ calculated for $\mathrm{C}_{13} \mathrm{H}_{25} \mathrm{NO}_{3}:[\mathrm{M}+\mathrm{H}]^{+}: 244.1907$, found: 244.1910. FTIR $\left(\mathrm{KBr}, \mathrm{cm}^{-1}\right)$ : 3626.51, $3416.38,3010.22,2998.12,1788.32,1651.23,1634.44$.
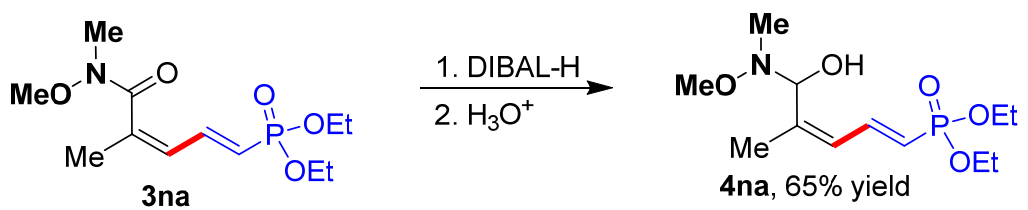

To a solution of diene $3 \mathrm{na}\left(29.1 \mathrm{mg}, 0.1 \mathrm{mmol}, 1.0\right.$ equiv.) in anhydrous $\mathrm{CH}_{2} \mathrm{Cl}_{2}(1 \mathrm{~mL})$ at $-78{ }^{\circ} \mathrm{C}$ was added DIBAL-H (1.0 M in heptane, $0.4 \mathrm{~mL}, 0.4 \mathrm{mmol}, 4.0$ equiv.) carefully over $20 \mathrm{~min}$. The resulting solution was stirred at $-78{ }^{\circ} \mathrm{C}$ for $2 \mathrm{~h}$ and quenched with saturated aqueous $\mathrm{NH}_{4} \mathrm{Cl}(0.1 \mathrm{~mL})$. The mixture was warmed to ambient temperature. The layer was separated and extracted with $\mathrm{CH}_{2} \mathrm{Cl}_{2}$ $(5 \mathrm{~mL} \times 3)$. The combined organic layers were washed with brine and dried over anhydrous $\mathrm{Na}_{2} \mathrm{SO}_{4}$. Concentration in vacuo and purification by column chromatography afforded the hemiaminal 4na as yellow oil (19 mg, 65\% yield). ${ }^{1} \mathrm{H}$ NMR $\left(500 \mathrm{MHz}, \mathrm{CDCl}_{3}\right): \delta=7.35-7.45(\mathrm{~m}, 1 \mathrm{H}), 6.03(\mathrm{~d}, J=11.5$ Hz, 1H), 5.55 (dd, $J=17.0 \mathrm{~Hz}, J=20.0 \mathrm{~Hz}, 1 \mathrm{H}), 3.98-4.04$ (m, 4H), 3.38 (s, 3H), 3.34 (brs, 1H), 2.49 (s, 3H), 1.89 (s, 3H), 1.34 (t, J = 7.0 Hz, 6H). ${ }^{13} \mathrm{C}$ NMR (125 MHz, $\left.\mathrm{CDCl}_{3}\right): \delta=144.06\left(\mathrm{~d}, J_{\mathrm{C}-\mathrm{P}}=6.8\right.$ $\mathrm{Hz}), 143.71,128.15\left(\mathrm{~d}, J_{\mathrm{C}-\mathrm{P}}=26.8 \mathrm{~Hz}\right), 115.63\left(\mathrm{~d}, J_{\mathrm{C}-\mathrm{P}}=190.0 \mathrm{~Hz}\right), 61.64\left(\mathrm{~d}, J_{\mathrm{C}-\mathrm{P}}=5.0 \mathrm{~Hz}\right), 60.86$, 59.69, 45.07, $23.81\left(\mathrm{~d}, J_{\mathrm{C}-\mathrm{P}}=5.0 \mathrm{~Hz}\right), 16.35\left(\mathrm{~d}, J_{\mathrm{C}-\mathrm{P}}=6.4 \mathrm{~Hz}\right) . \mathrm{HR}-\mathrm{MS}(\mathrm{ESI}): \mathrm{m} / \mathrm{z}$ calculated for $\mathrm{C}_{12} \mathrm{H}_{24} \mathrm{NO}_{5} \mathrm{P}:[\mathrm{M}+\mathrm{H}]^{+}:$293.1392, found: 293.1391. FTIR $\left(\mathrm{KBr}, \mathrm{cm}^{-1}\right): 3626.51,3416.38,2348.23$, $1688.32,1634.44,1661.66,1384.32,1019.70$. 


\section{References}

1. K. Meng, J. Zhang, F. Li, Z. Lin, K. Zhang, G. Zhong, Org. Lett. 2017, 19, 2498.

2. C. Feng, D. Feng, T. P. Loh, Chem. Commun. 2015, 51, 342. 


\section{NMR Spectra}<smiles>CCOP(=O)(/C=C/C=C(/C)C(=O)N(C)C)OCC</smiles>

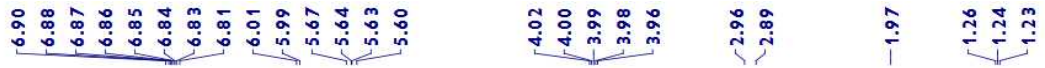

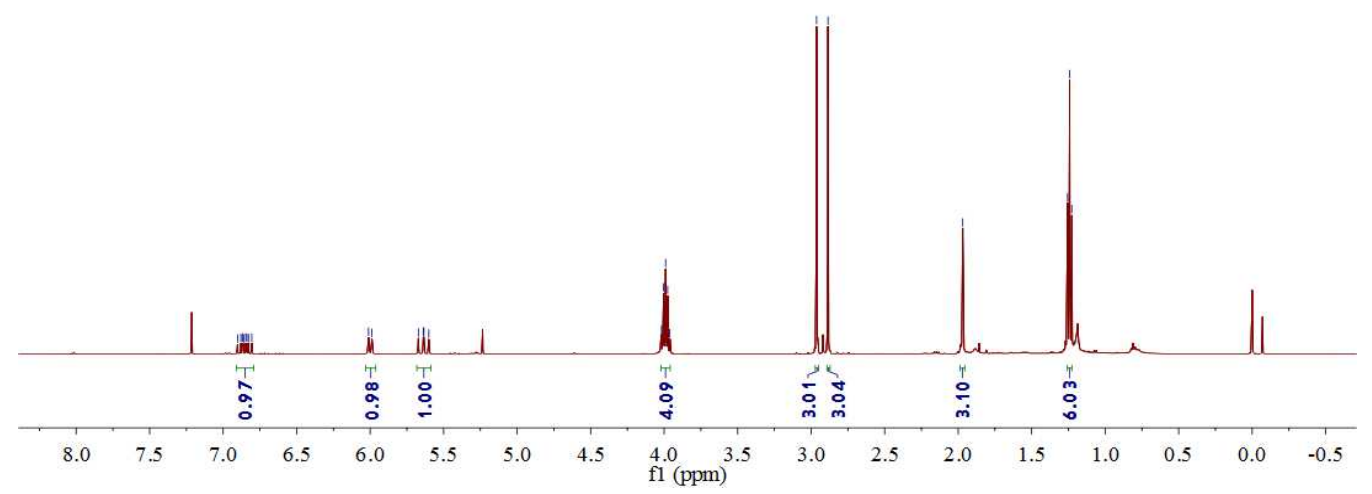

草 过

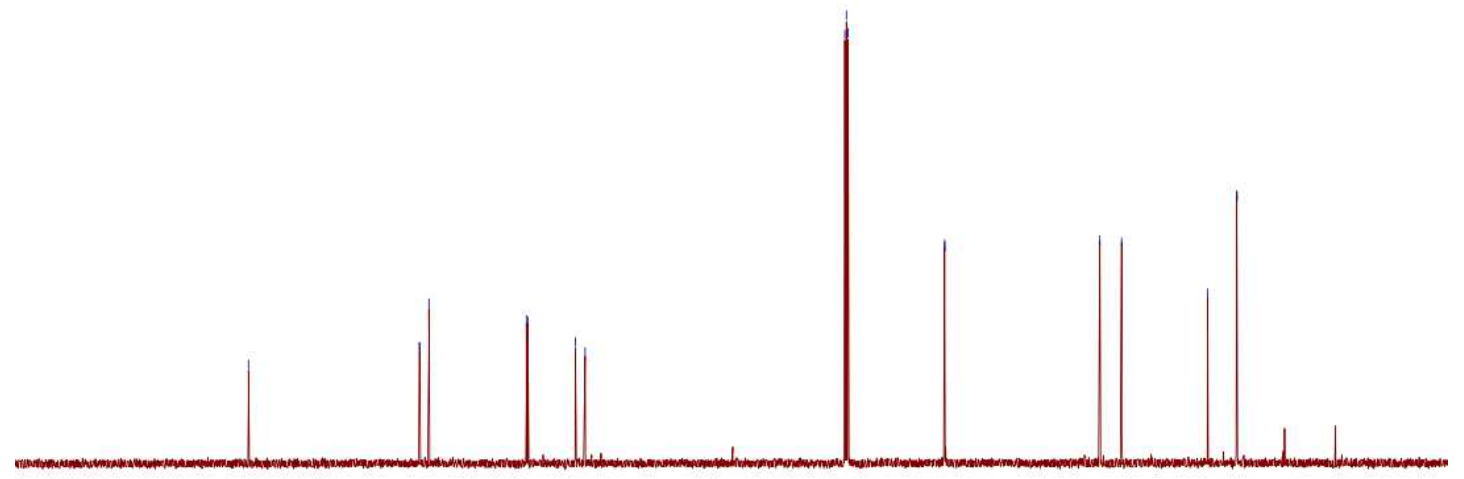

$\begin{array}{llllllllllllllllllllll}200 & 190 & 180 & 170 & 160 & 150 & 140 & 130 & 120 & 110 & 100 & 90 & 80 & 70 & 60 & 50 & 40 & 30 & 20 & 10 & 0 & -10\end{array}$ 
<smiles>CCOP(=O)(/C=C/C=C(/C(=O)N(C)C)c1ccccc1)OCC</smiles>

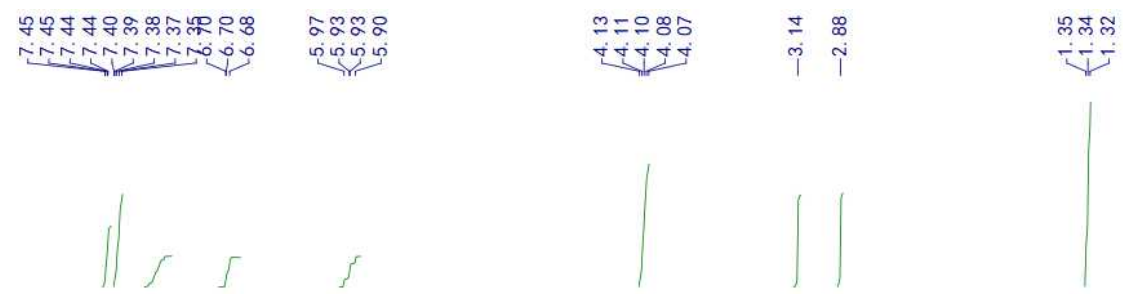

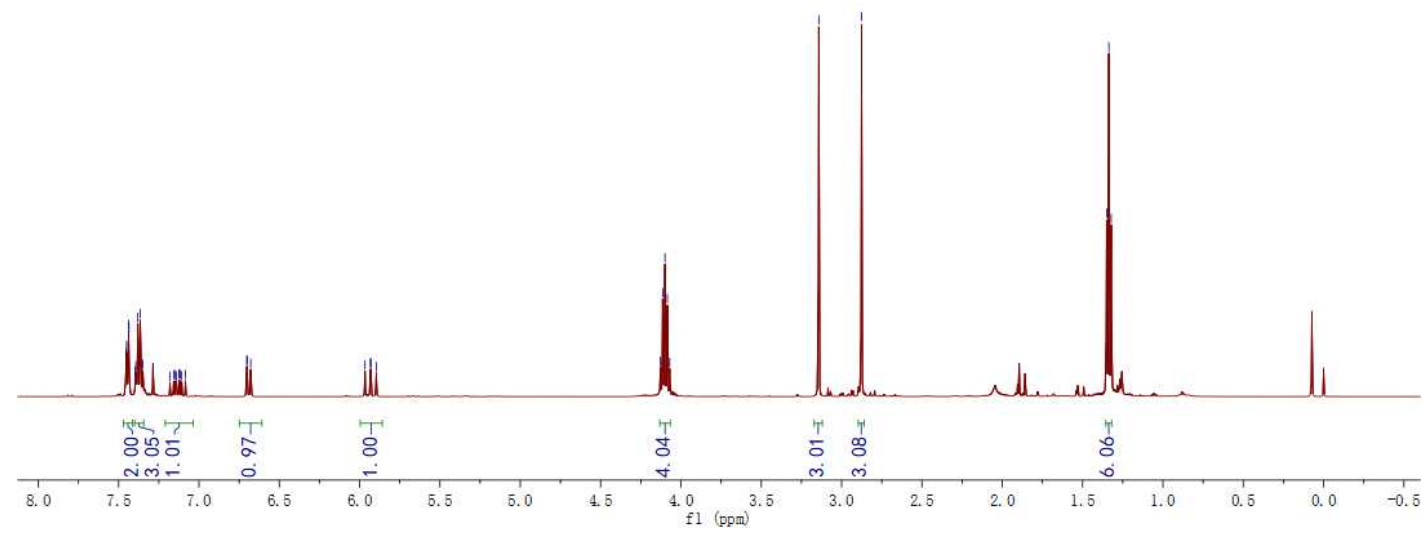

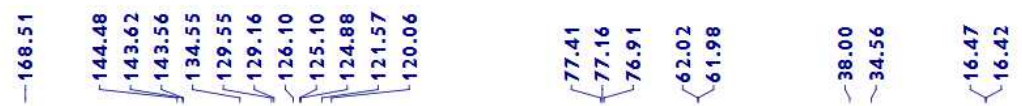

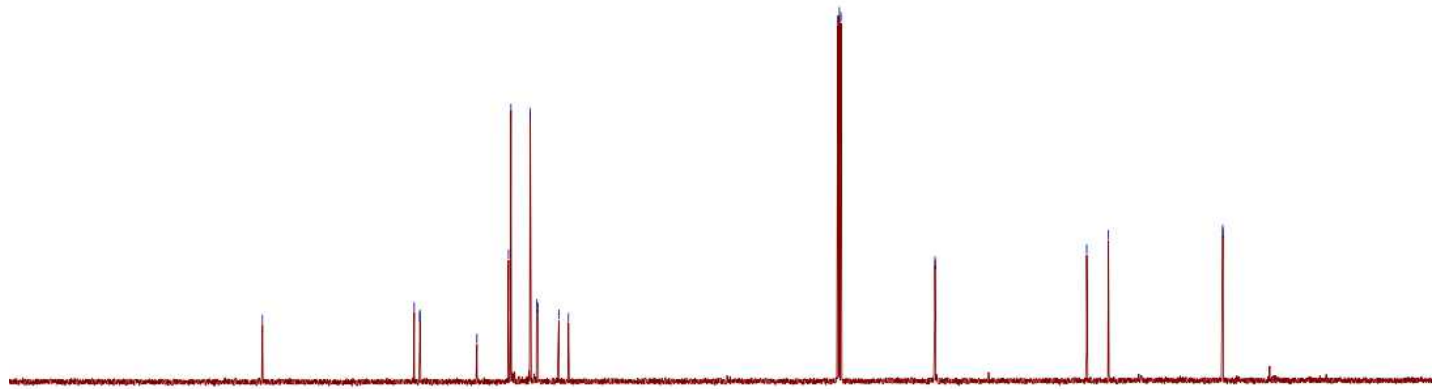

$\begin{array}{lllllllllllllllllllllll}200 & 190 & 180 & 170 & 160 & 150 & 140 & 130 & 120 & 110 & 100 & 90 & 80 & 70 & 60 & 50 & 40 & 30 & 20 & 10 & 0 & -10\end{array}$ 
<smiles>CCOP(=O)(/C=C/C=C(/C(=O)N(C)C)c1ccc(Cl)cc1)OCC</smiles>

(3ca)

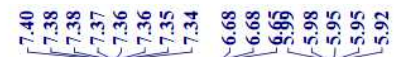

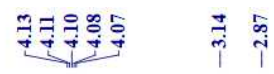

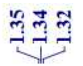

1,1
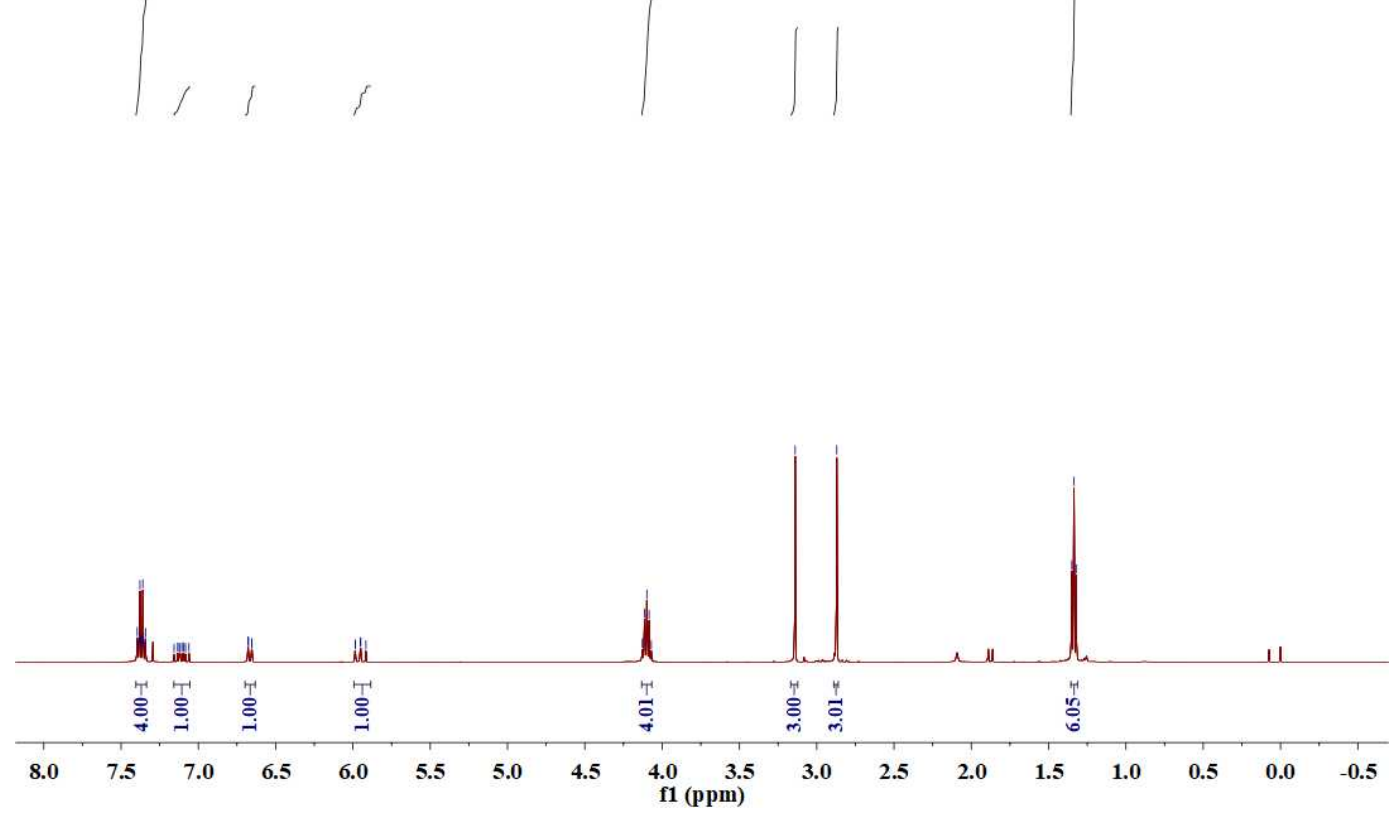

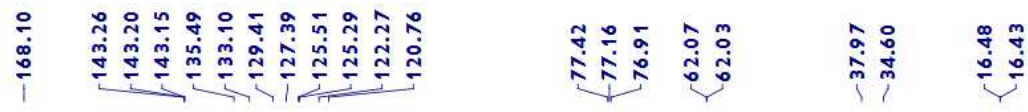

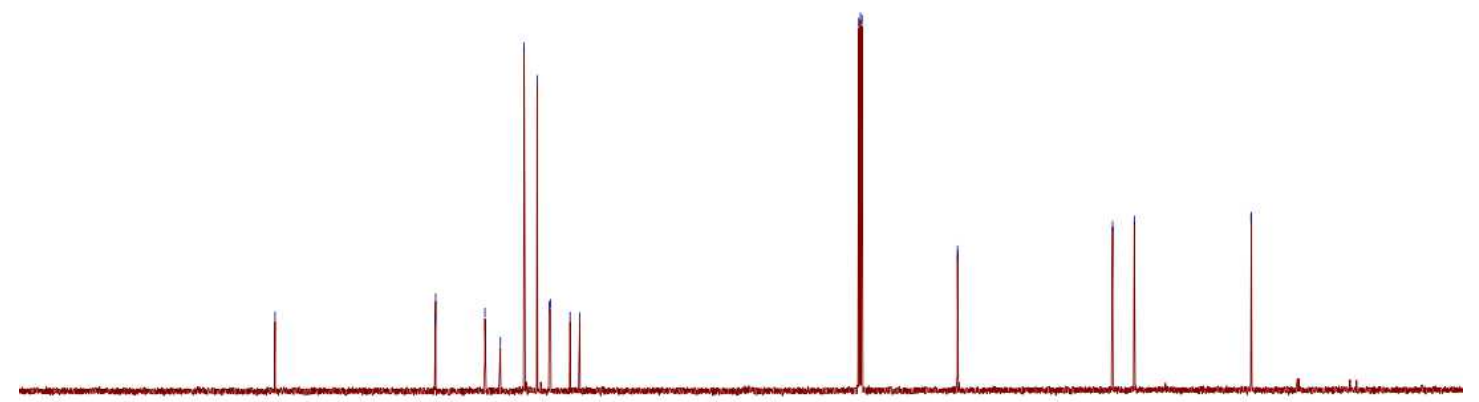

$\begin{array}{llllllllllllllllllllll}200 & 190 & 180 & 170 & 160 & 150 & 140 & 130 & 120 & 110 & \begin{array}{l}100 \\ \mathrm{fl}(\mathrm{ppm})\end{array} & 80 & 70 & 60 & 50 & 40 & 30 & 20 & 10 & 0 & -10\end{array}$ 
<smiles>CCOP(=O)(/C=C/C=C(/C(=O)N(C)C)c1ccc(F)cc1)OCC</smiles>

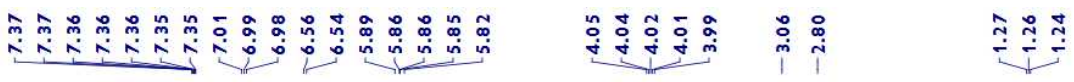
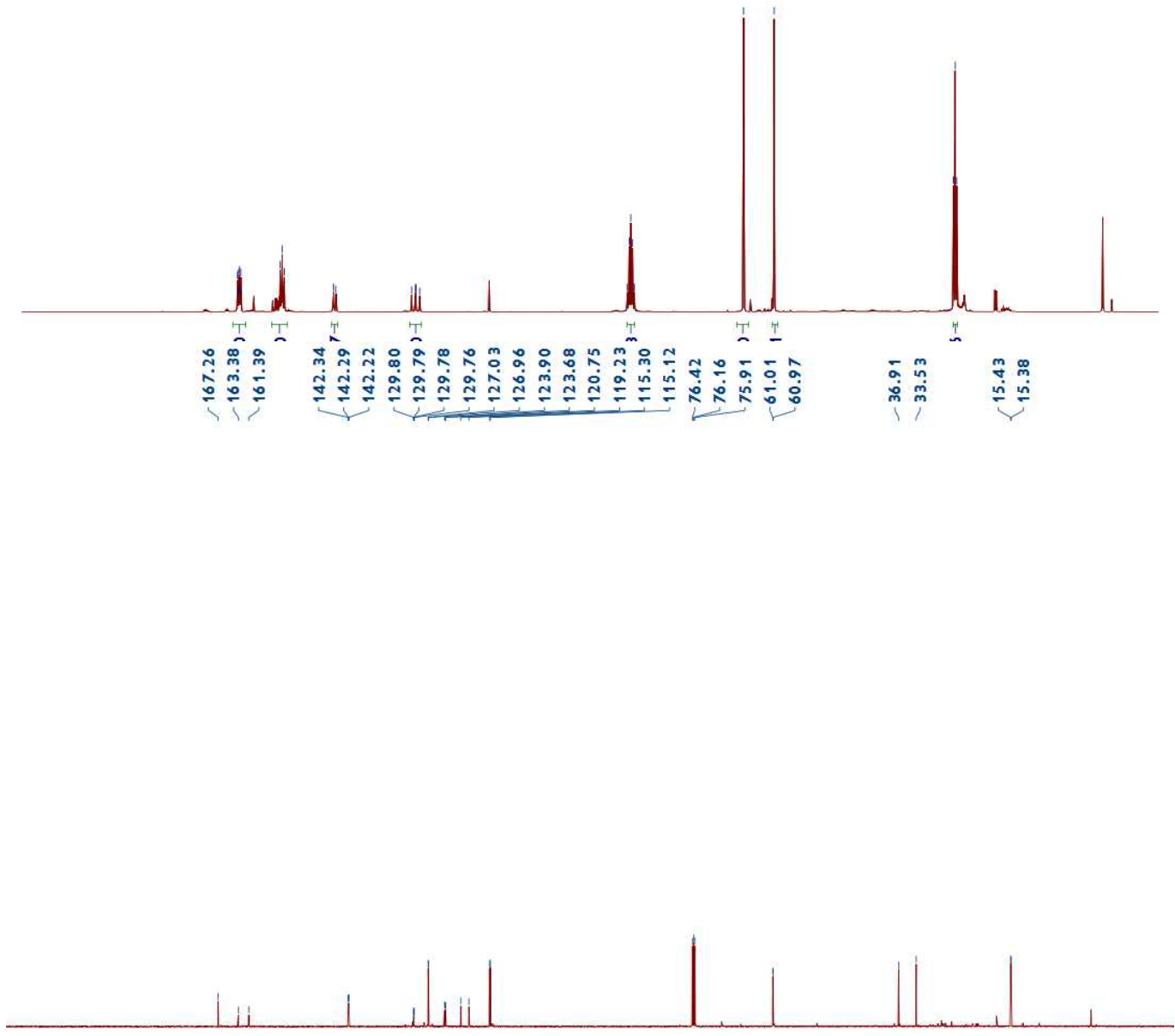

$\begin{array}{llllllllllllllllllllll}200 & 190 & 180 & 170 & 160 & 150 & 140 & 130 & 120 & 110 & 100 & 90 & 80 & 70 & 60 & 50 & 40 & 30 & 20 & 10 & 0 & -10\end{array}$ 


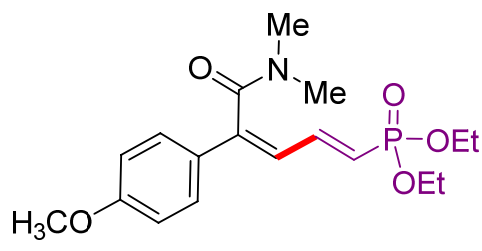

(3ea)

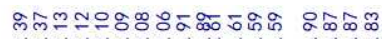

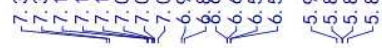

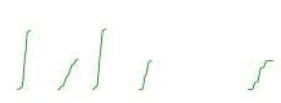

ஜூల్ల

$\iiint$

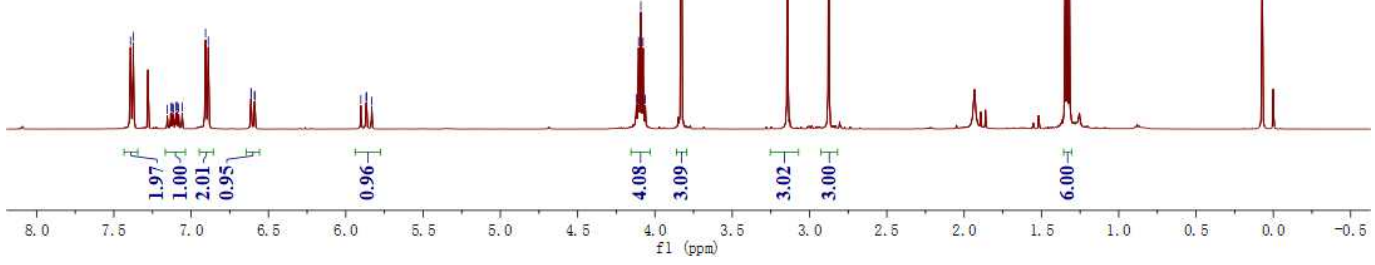

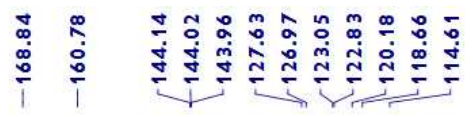

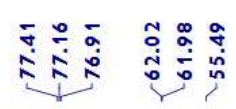

量

管敦
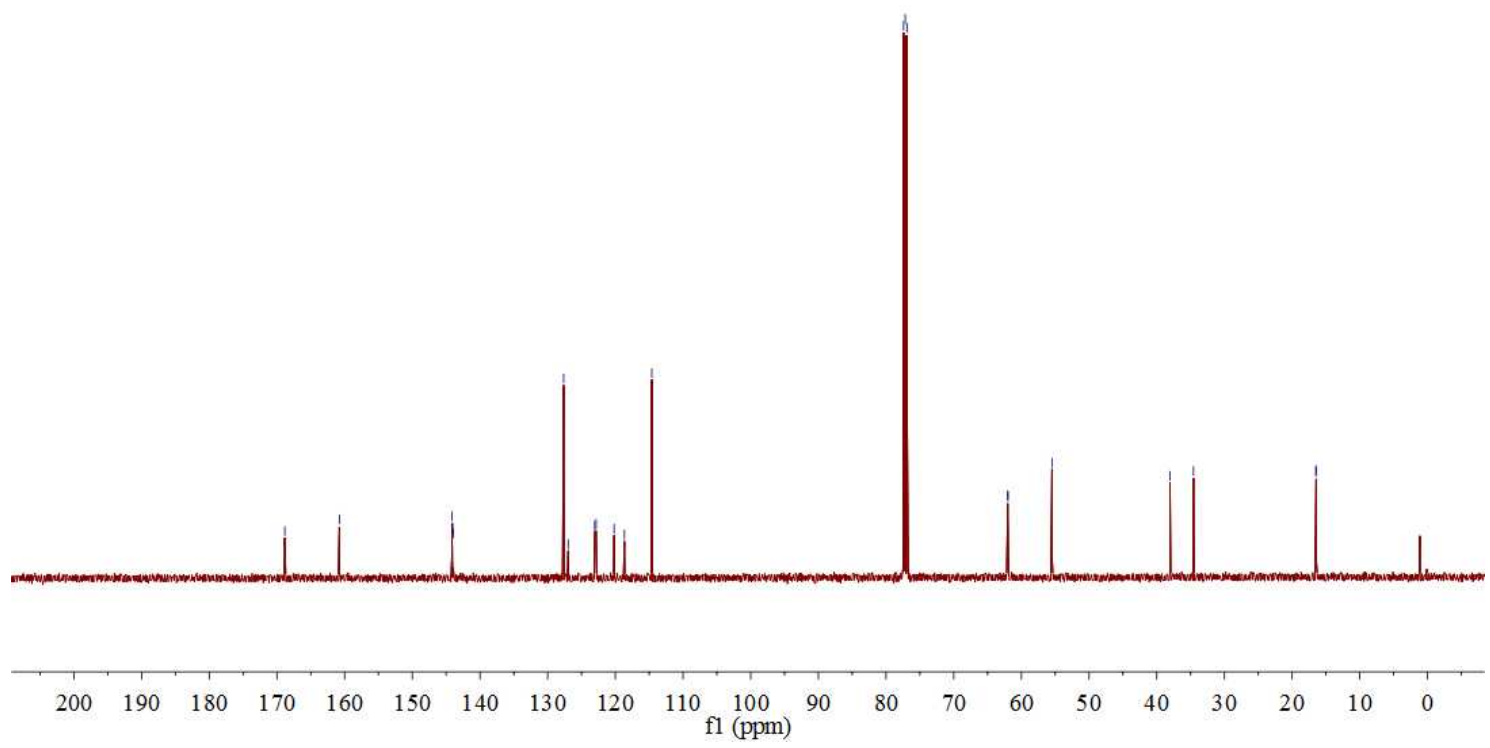

25 
<smiles>CCOP(=O)(/C=C/C=C(\C(=O)N(C)C)c1ccc(C(F)(F)F)cc1)OCC</smiles>

(3fa)
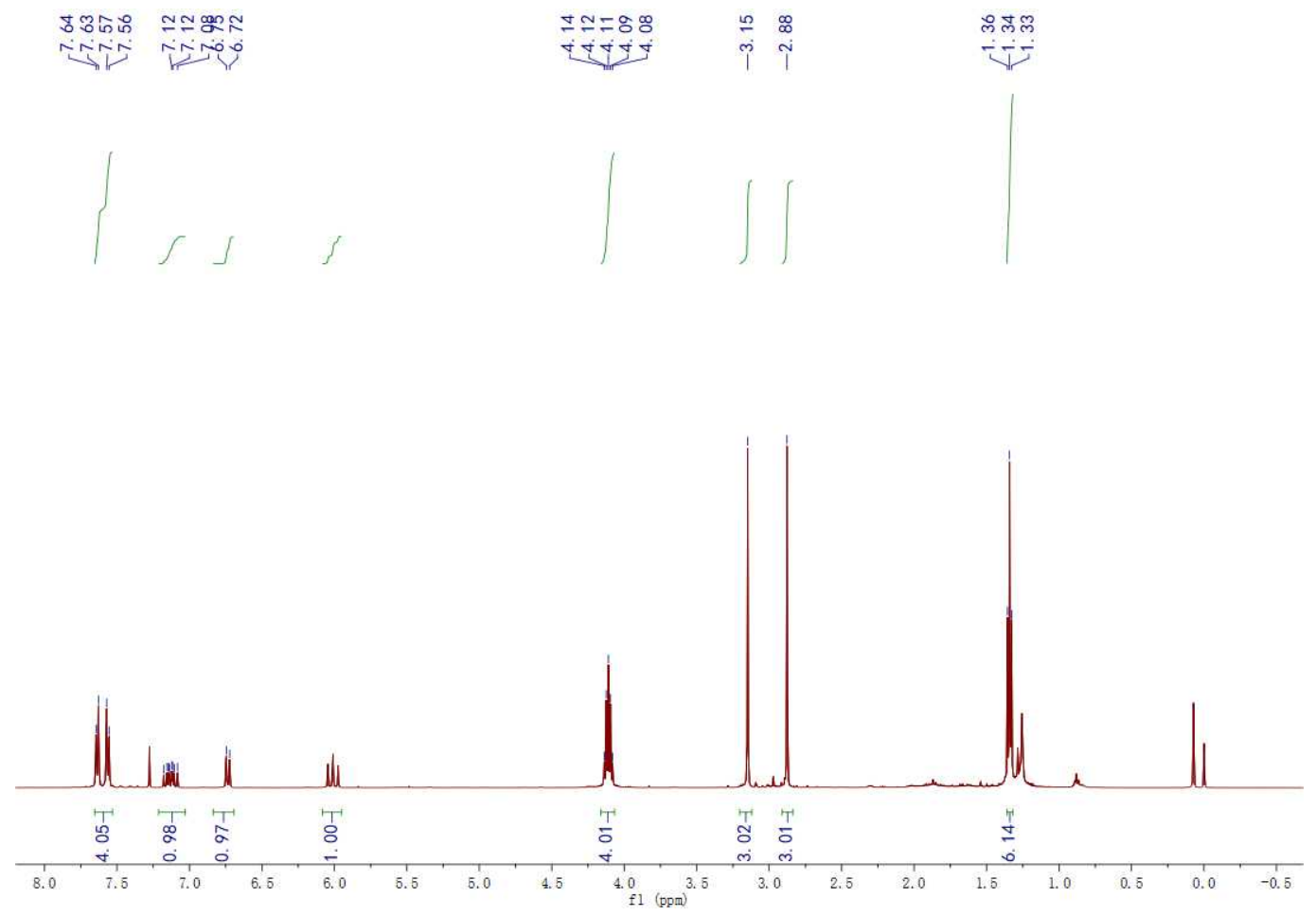

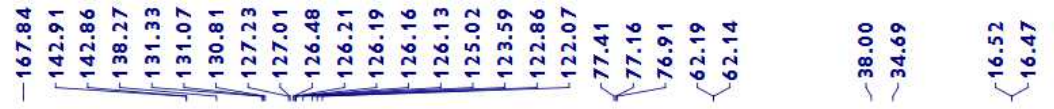

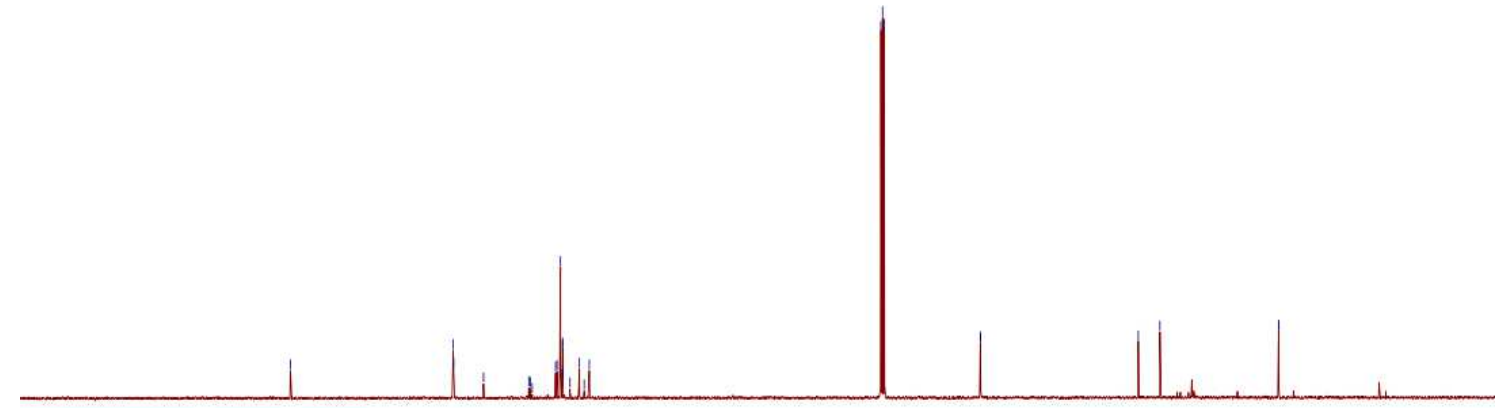

$\begin{array}{llllllllllllllllllllll}200 & 190 & 180 & 170 & 160 & 150 & 140 & 130 & 120 & 110 & 100 & 90 & 80 & 70 & 60 & 50 & 40 & 30 & 20 & 10 & 0 & -10\end{array}$ 
<smiles>CCOP(=O)(/C=C/C=C(/C(=O)N(C)C)c1cccc2ccccc12)OCC</smiles>

(3ga)

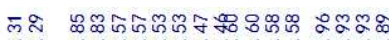

in virinininiogio niving

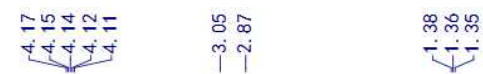

$51 / 11$

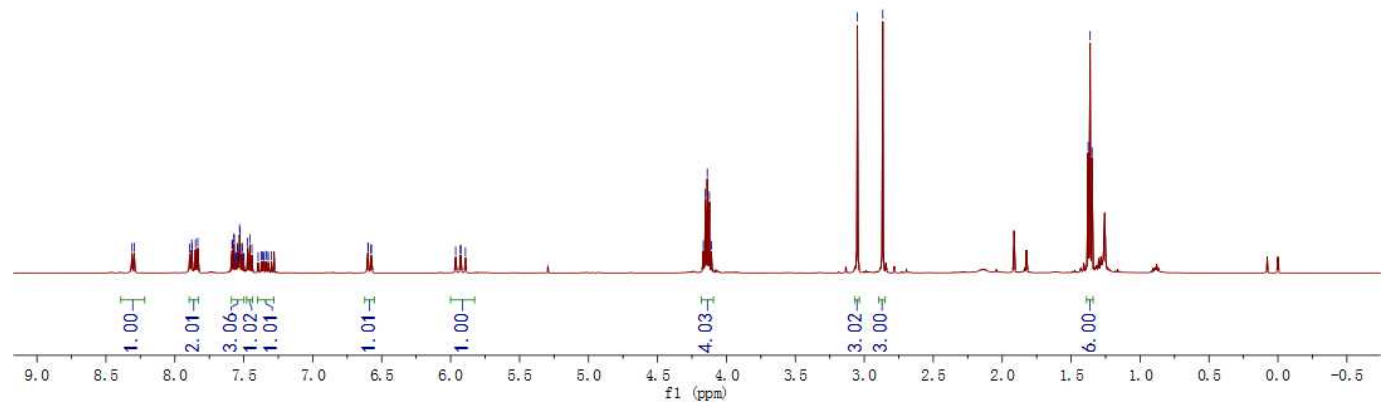

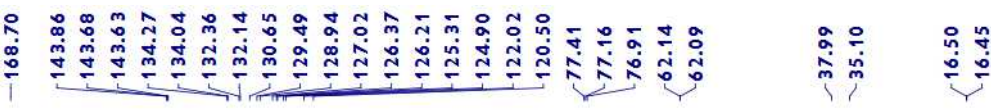

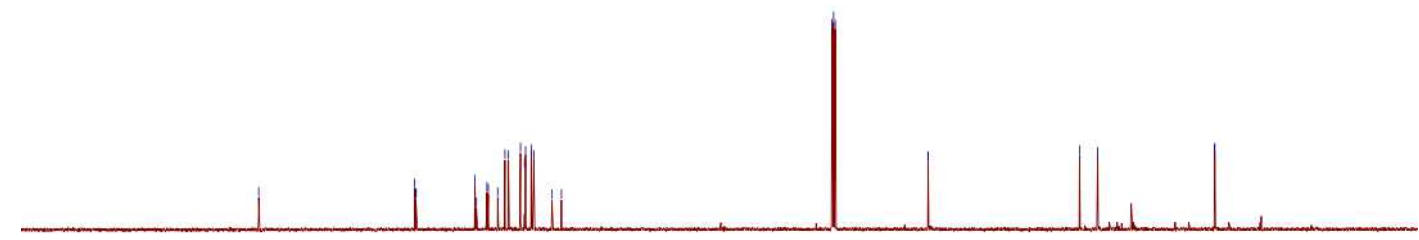

$\begin{array}{llllllllllllllllllllll}200 & 190 & 180 & 170 & 160 & 150 & 140 & 130 & 120 & 110 & 100 & 90 & 80 & 70 & 60 & 50 & 40 & 30 & 20 & 10 & 0 & -10\end{array}$ 
<smiles>CCOP(=O)(/C=C/C1=C(C(=O)N(C)C)CCCC1)OCC</smiles>

(3ha)

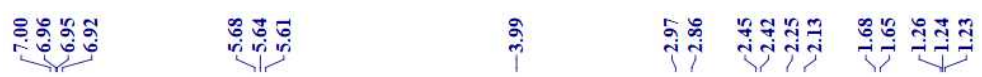

r $|\|, 1| \mid$

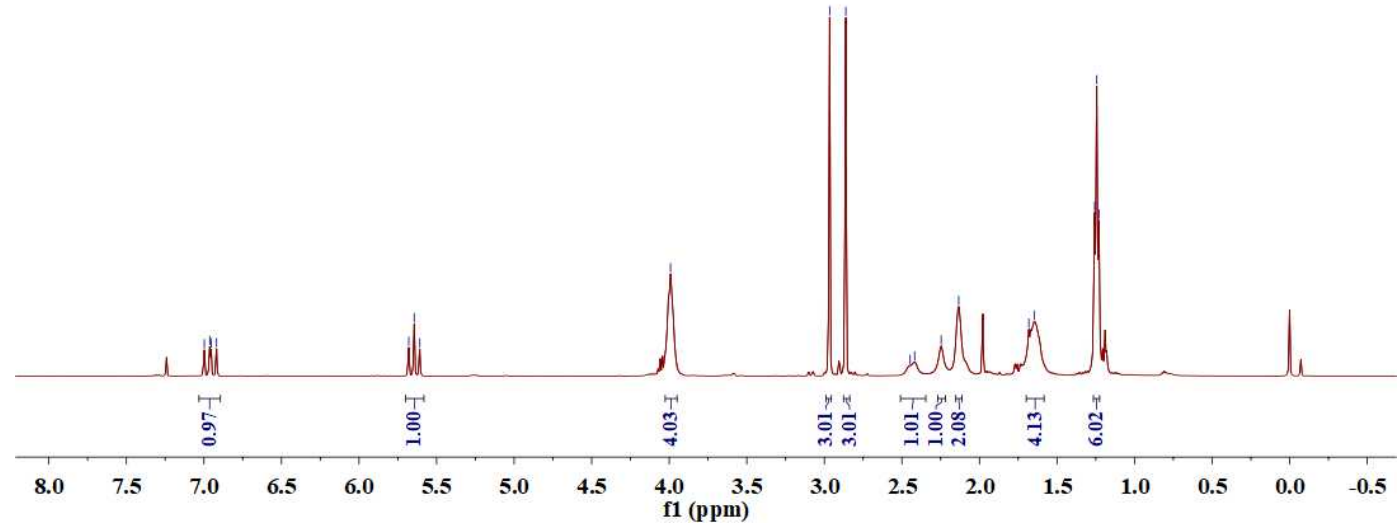

染

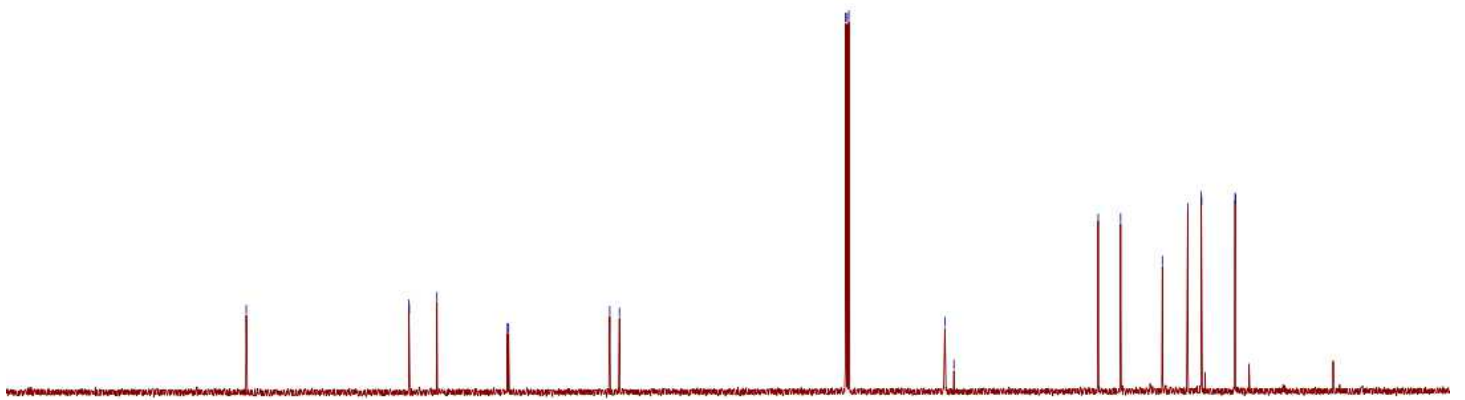

$\begin{array}{llllllllllllllllllllll}200 & 190 & 180 & 170 & 160 & 150 & 140 & 130 & 120 & 110 & 100 & 90 & 80 & 70 & 60 & 50 & 40 & 30 & 20 & 10 & 0 & -10\end{array}$ 


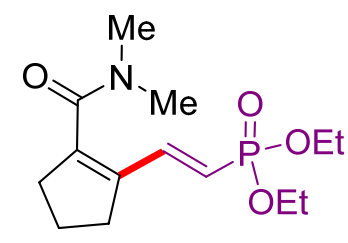

(3ia)
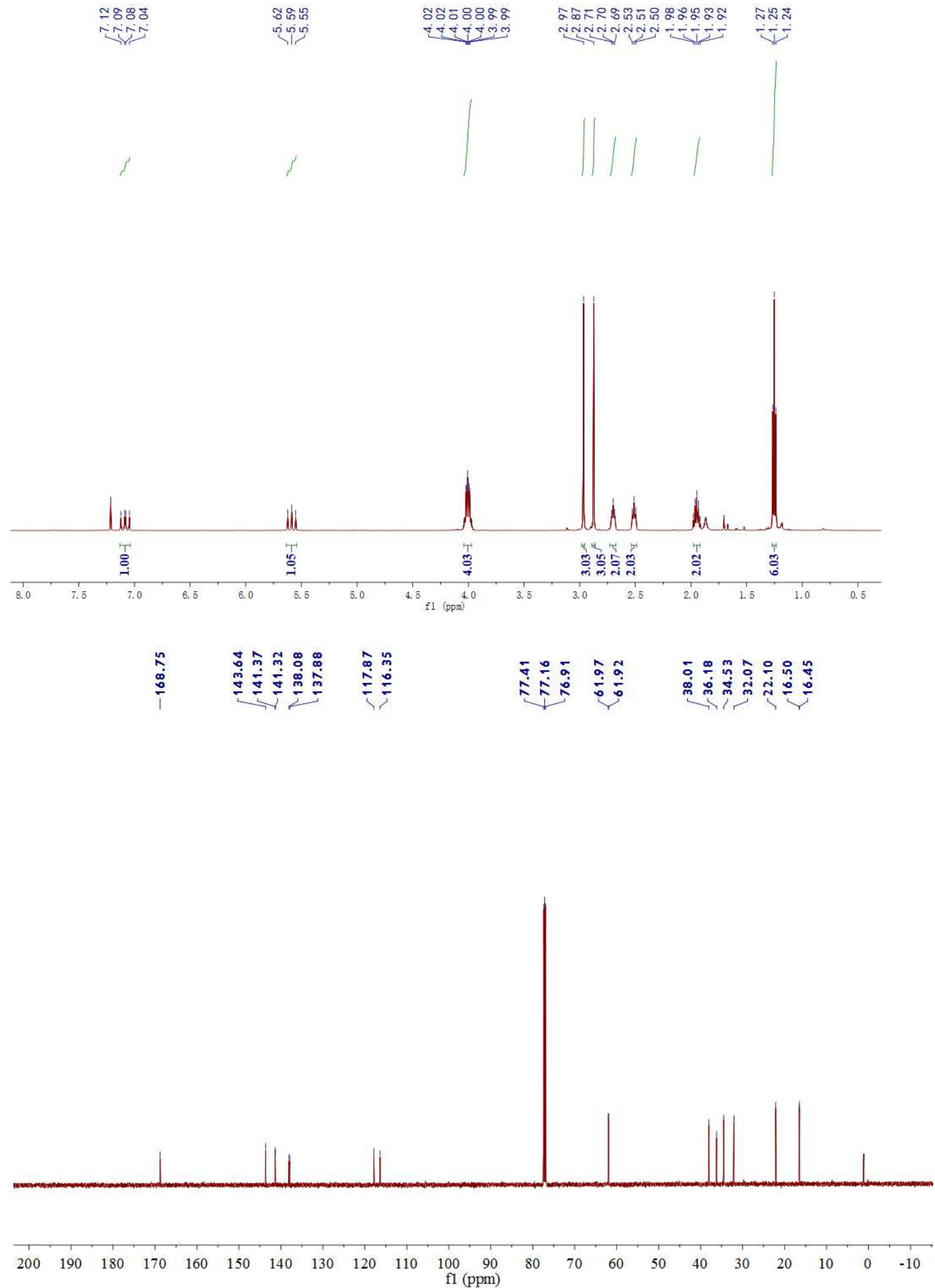
<smiles>CCCC(=CC=CP(=O)(OCC)OCC)C(=O)N(C)C</smiles>

(3ja)
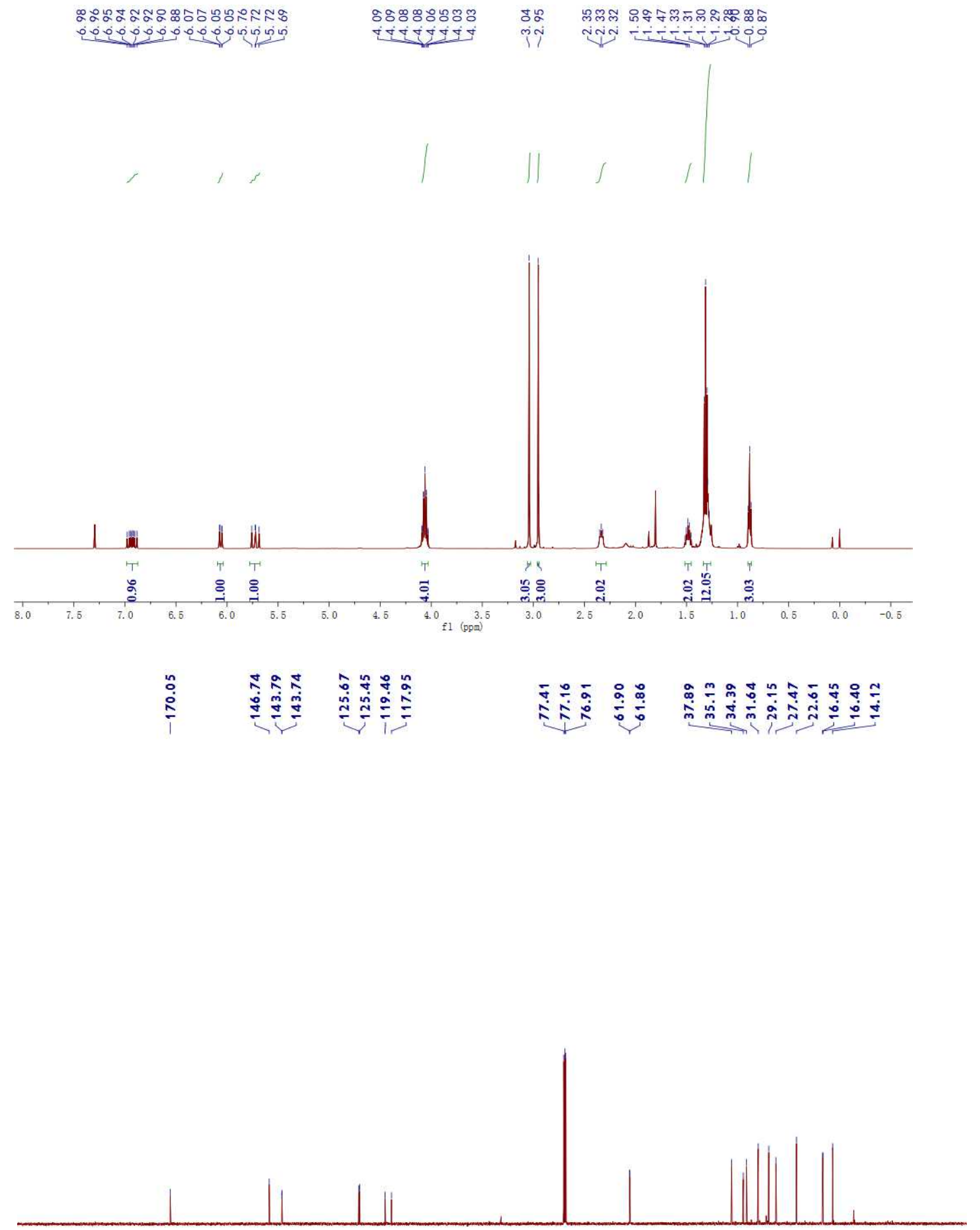

$\begin{array}{llllllllllllllllllllll}200 & 190 & 180 & 170 & 160 & 150 & 140 & 130 & 120 & 110 & 100 & 90 & 80 & 70 & 60 & 50 & 40 & 30 & 20 & 10 & 0 & -10\end{array}$ 
<smiles>CCOP(=O)(/C=C/C=C(/C)C(=O)N1CCCC1)OCC</smiles>

(3ka)

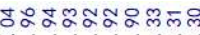
ㄴ-T-Z-
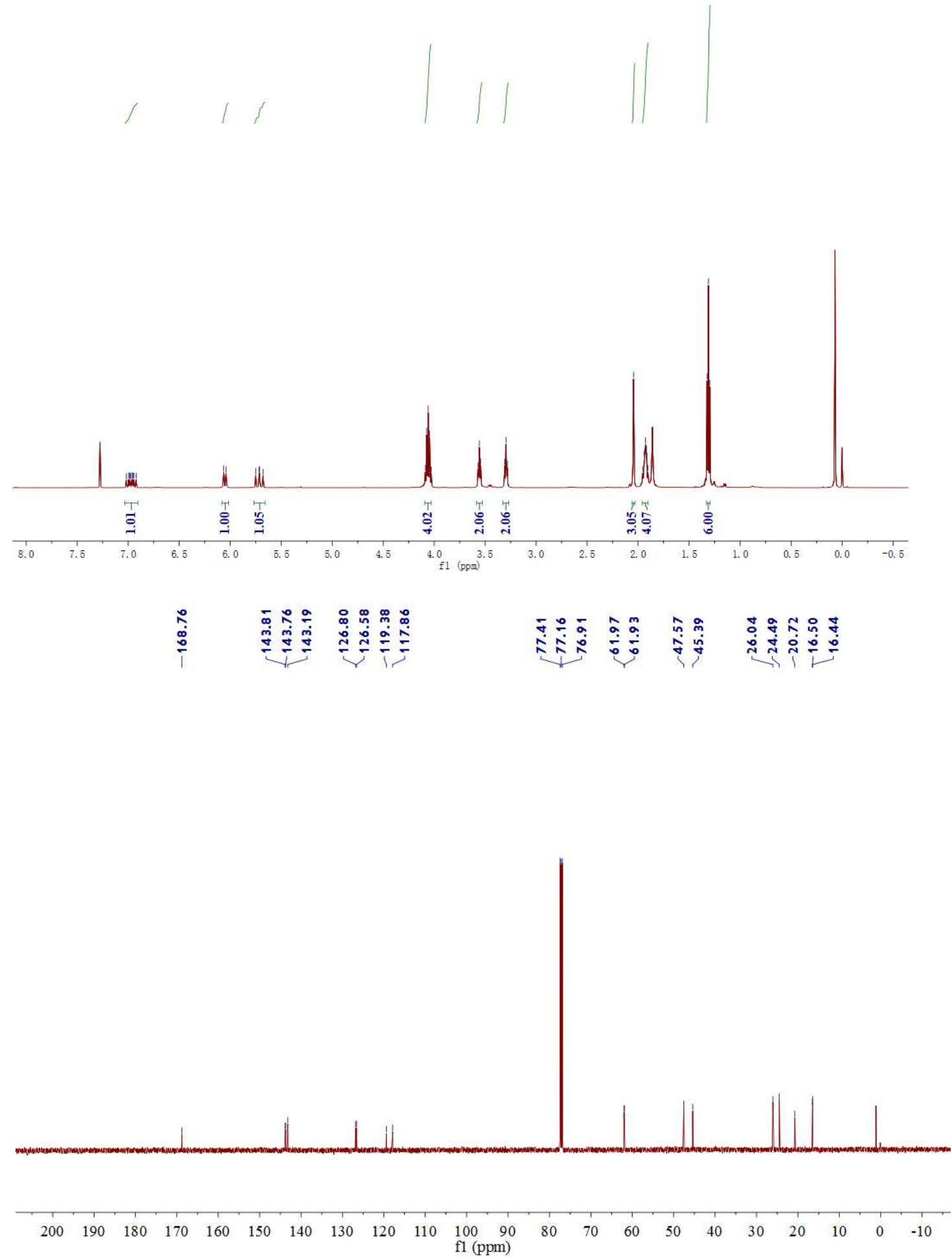
<smiles>CCOP(=O)(/C=C/C=C(/C)C(=O)N1CCCCC1)OCC</smiles>
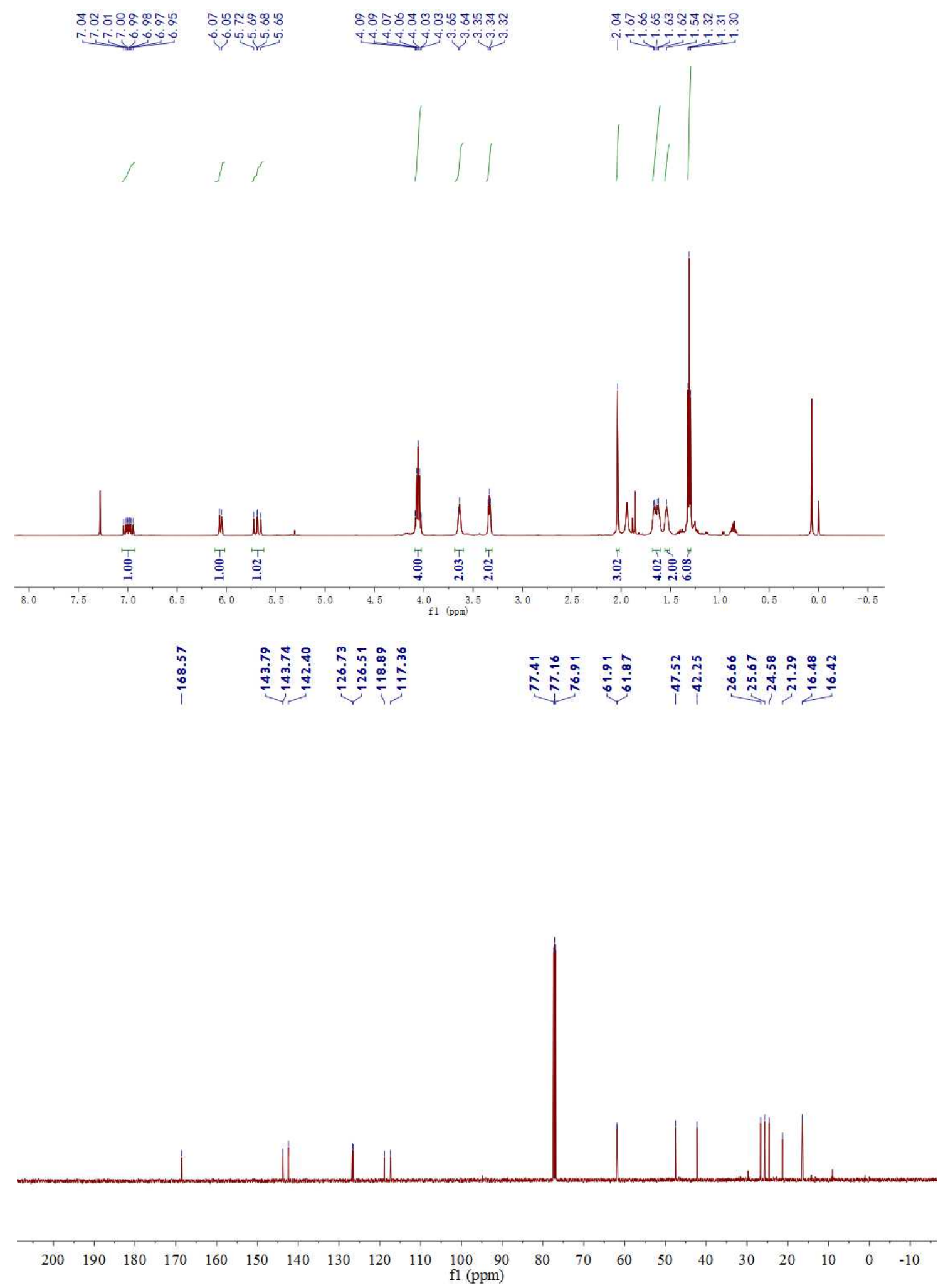
<smiles>CCOP(=O)(/C=C/C=C(/C)C(=O)N(CC)CC)OCC</smiles>

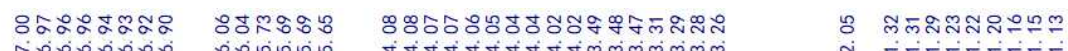

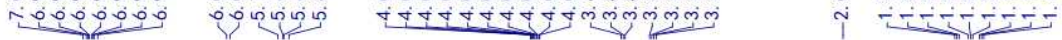

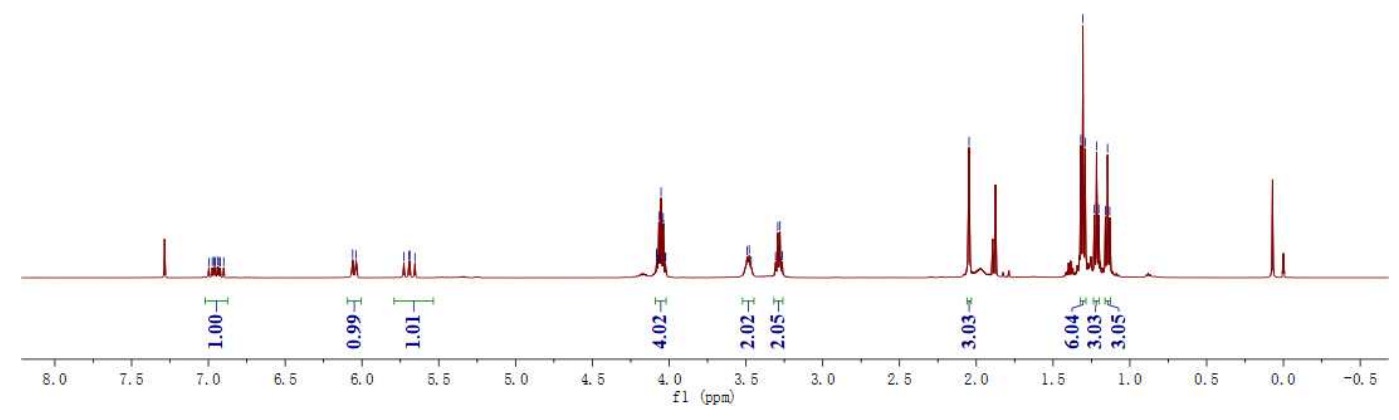

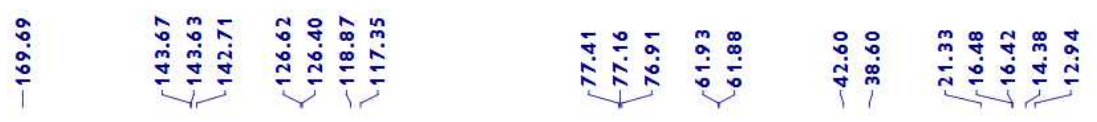

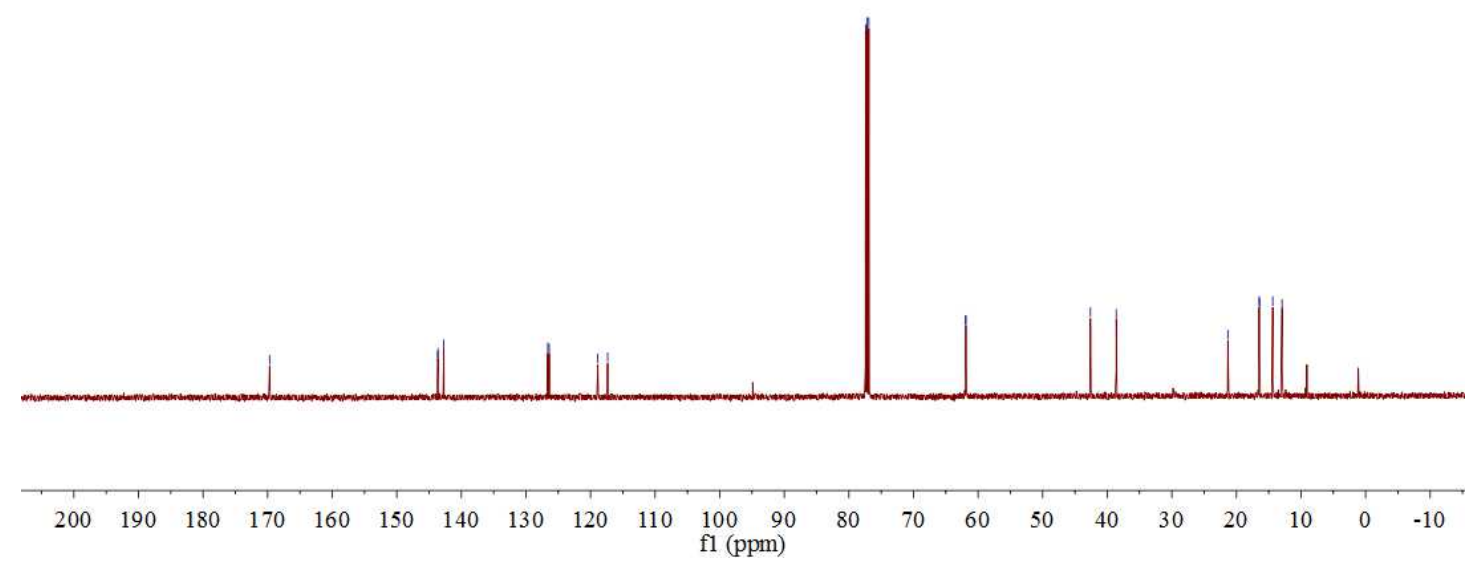


<smiles>CCOP(=O)(/C=C/C=C(/C)C(=O)N(C)OC)OCC</smiles>
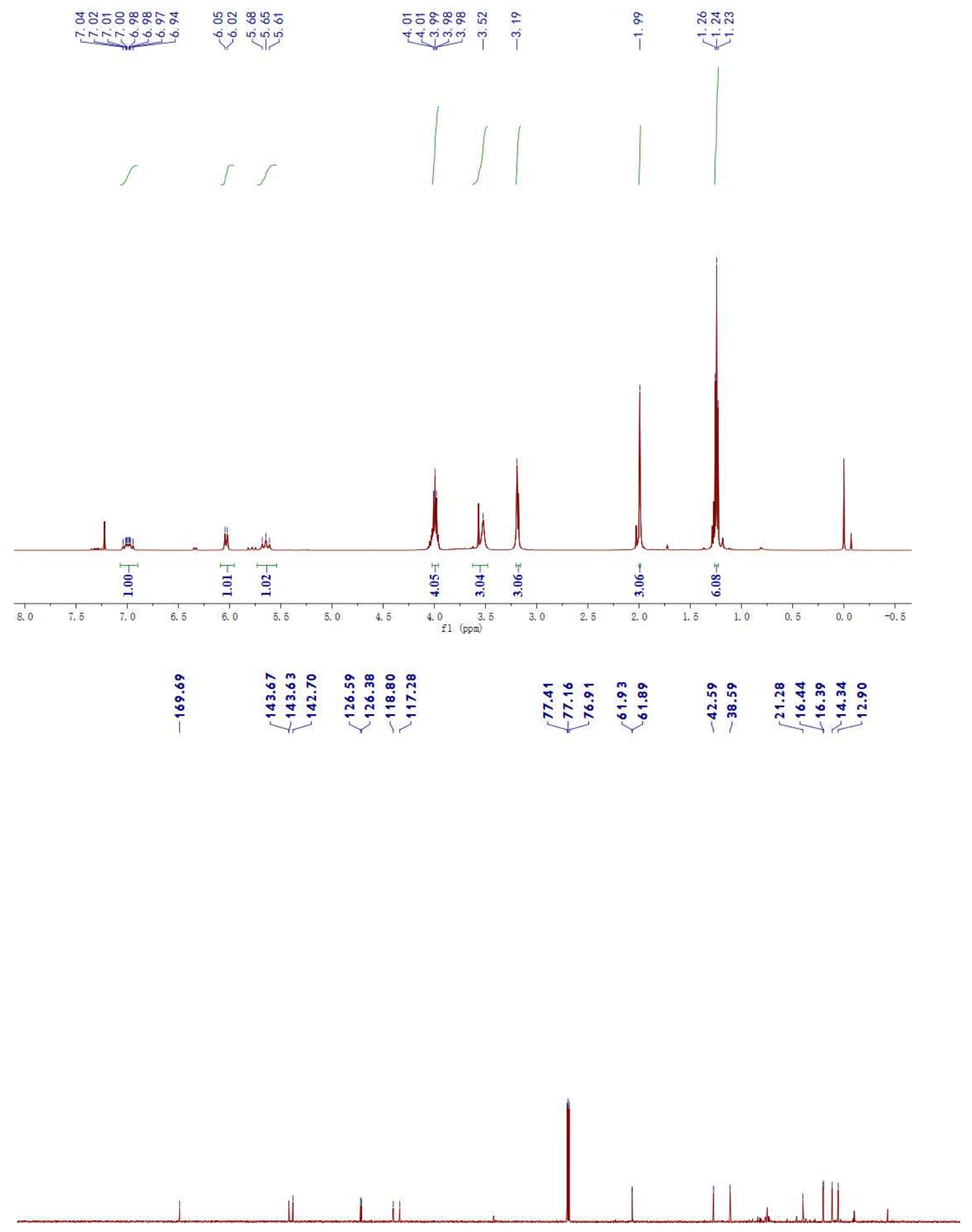

$\begin{array}{llllllllllllllllllllll}200 & 190 & 180 & 170 & 160 & 150 & 140 & 130 & 120 & 110 & 100 & 90 & 80 & 70 & 60 & 50 & 40 & 30 & 20 & 10 & 0 & -10\end{array}$ 
<smiles>CCOP(=O)(/C=C/C=C(/C)C(=O)N[Na])OCC</smiles>
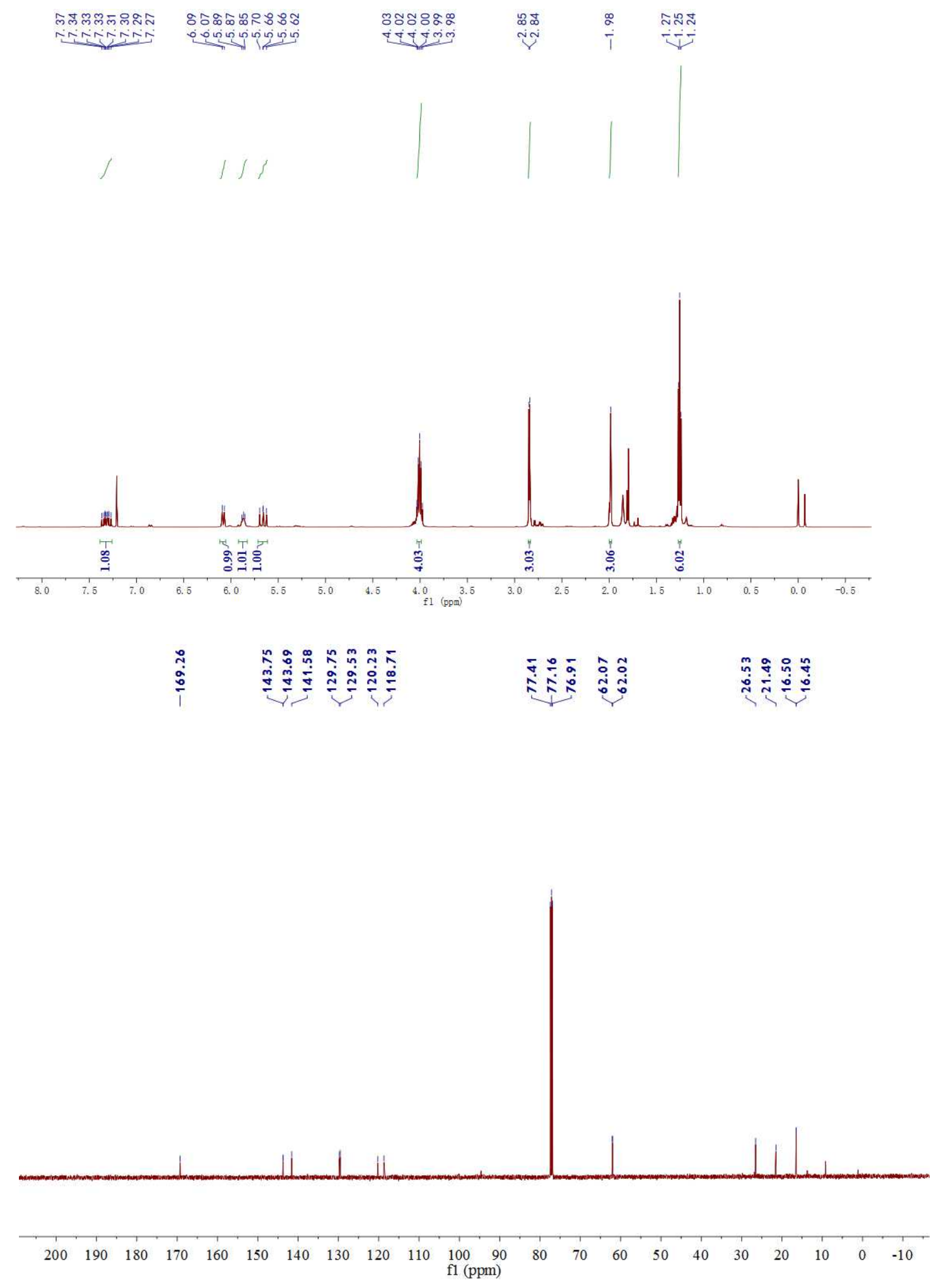
<smiles>C/C(=C/C=C/S(=O)(=O)c1ccccc1)C(=O)N(C)C</smiles>

(3ab)

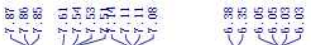

ริ)
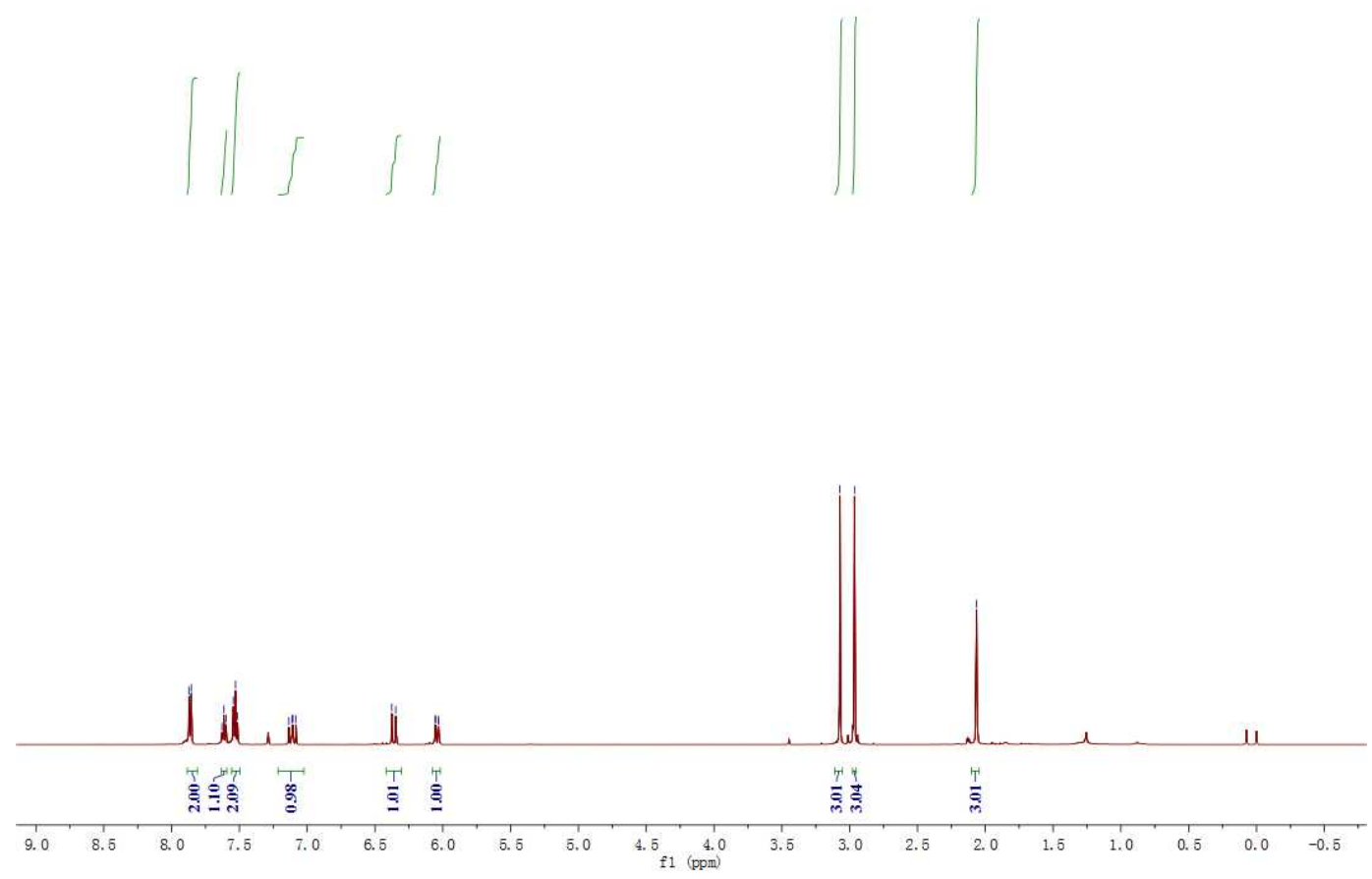

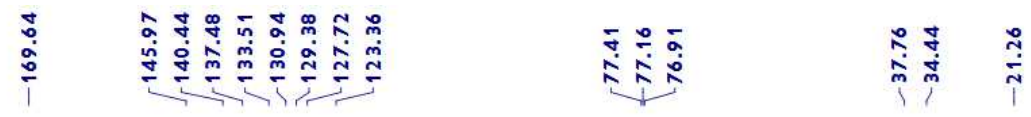

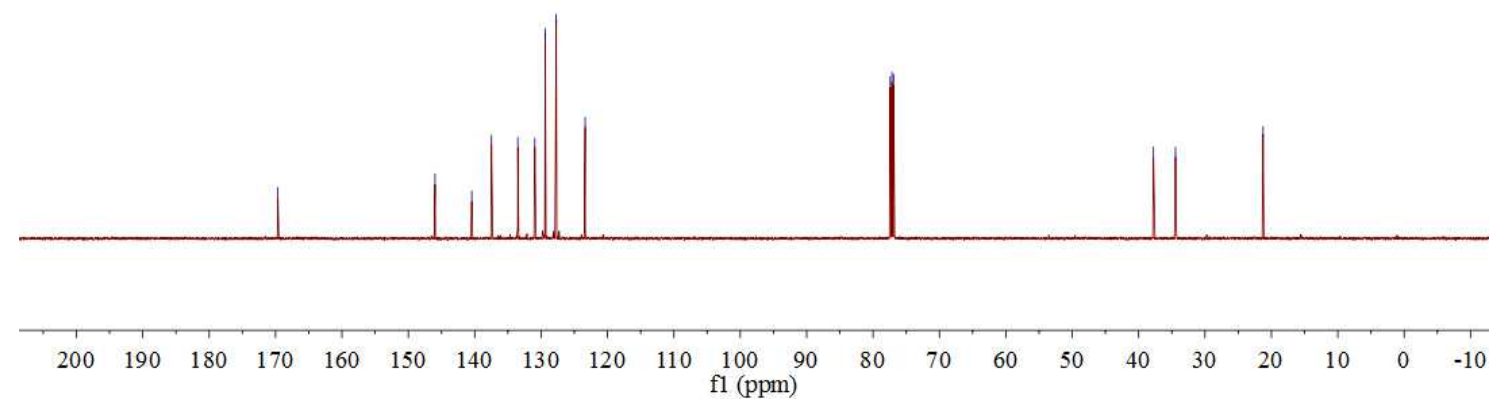


<smiles>CN(C)C(=O)/C(=C\C=C\S(=O)(=O)c1ccccc1)c1ccccc1</smiles>

(3bb)

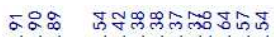

in ivininio

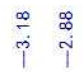
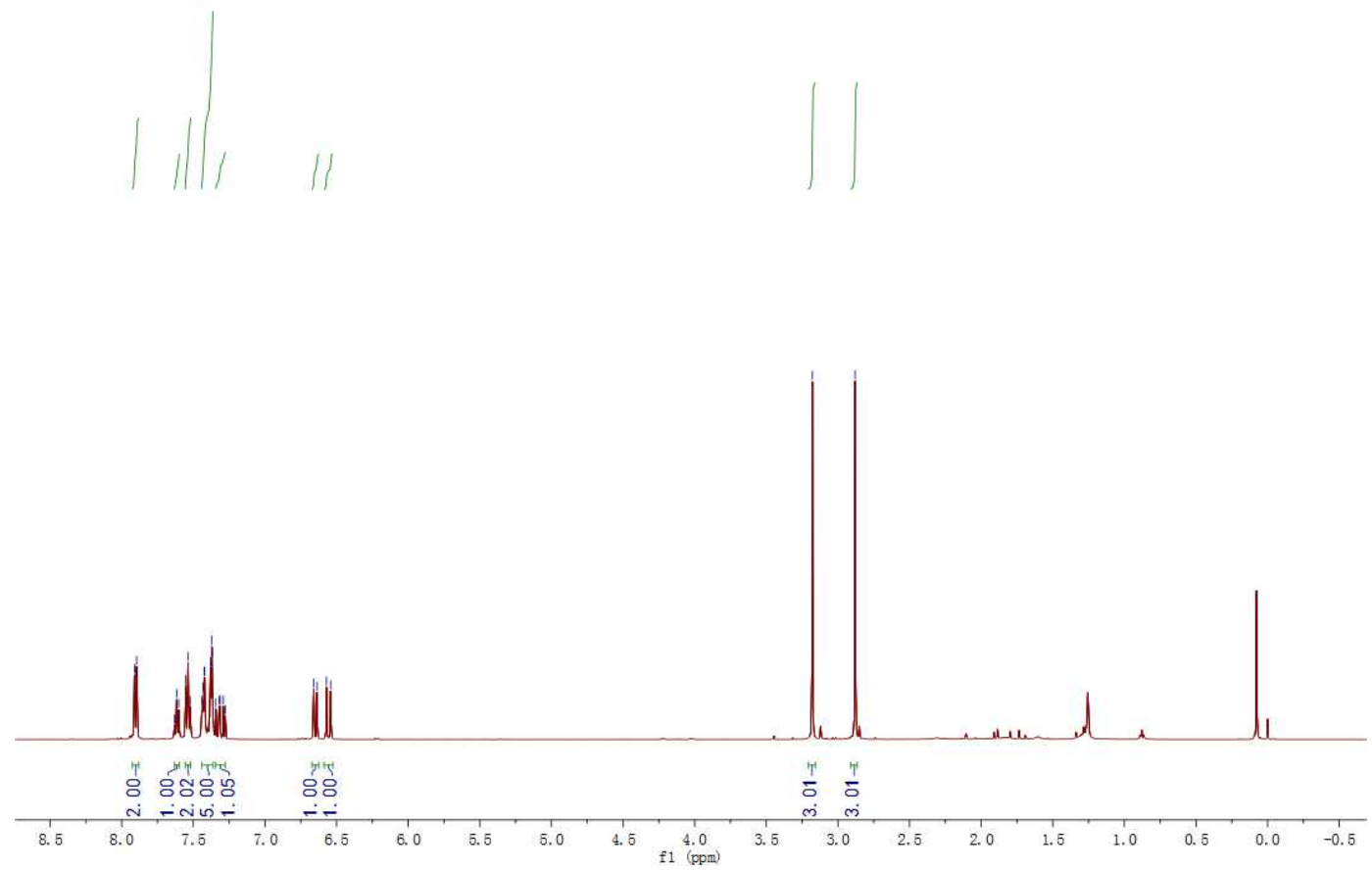

ปั

i

平率全

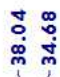

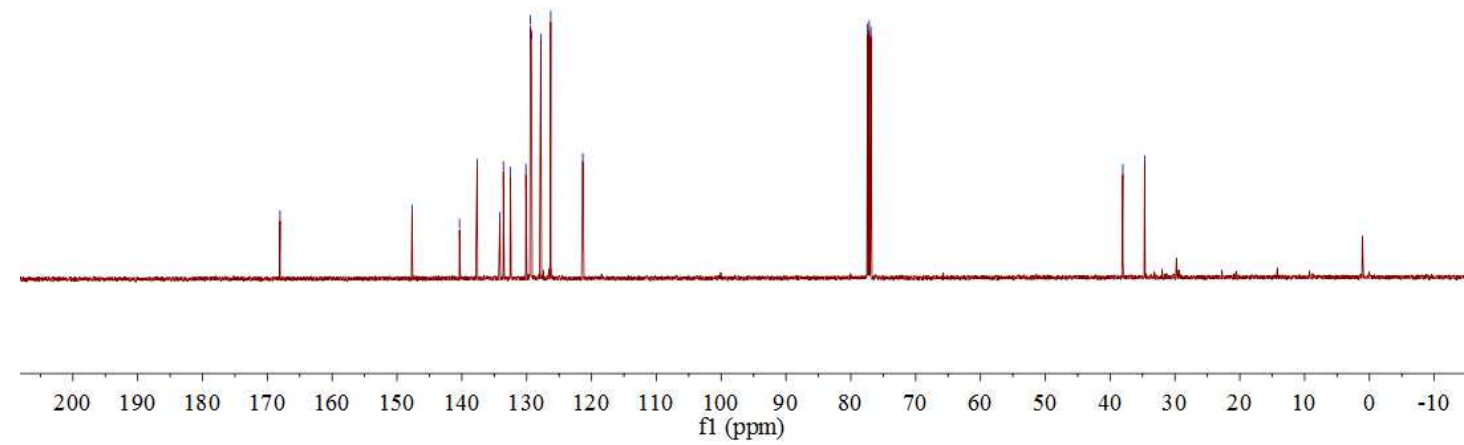


<smiles>CCCC(C)=C(CC=CS(=O)(=O)c1ccccc1)C(=O)N(C)C</smiles>

(3jb)

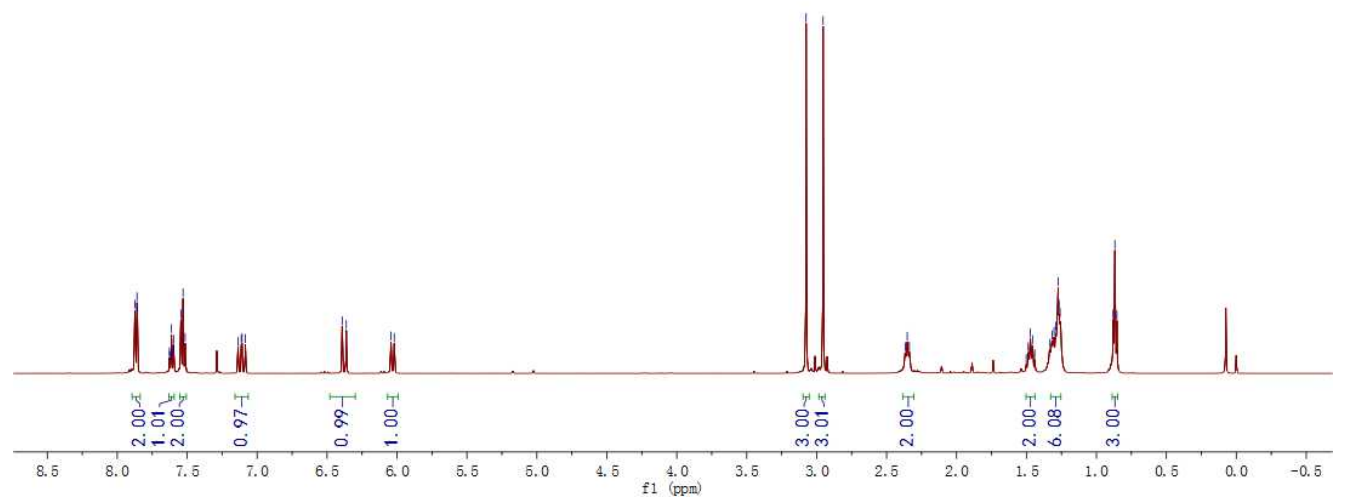

施

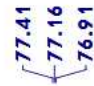

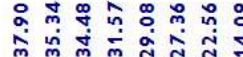

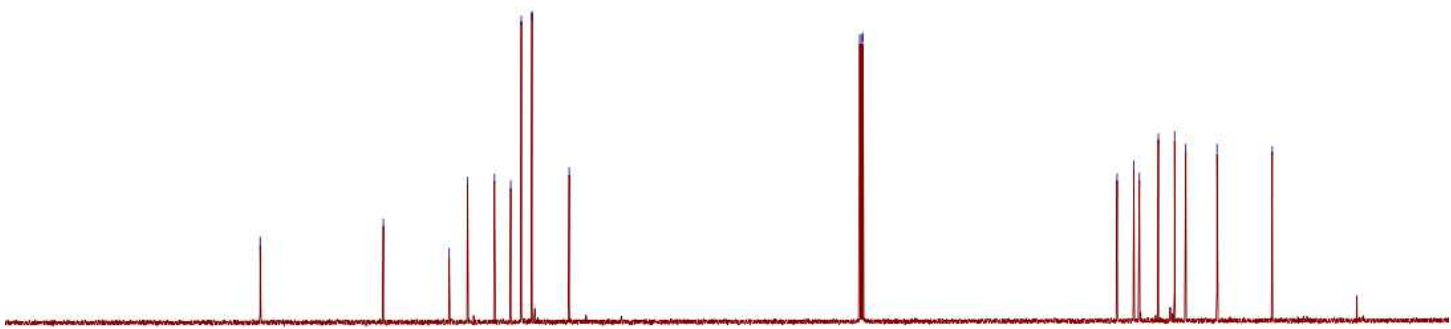

$\begin{array}{llllllllllllllllllllll}200 & 190 & 180 & 170 & 160 & 150 & 140 & 130 & 120 & 110 & 100 & 90 & 80 & 70 & 60 & 50 & 40 & 30 & 20 & 10 & 0 & -10\end{array}$ 
<smiles>CCOC(=O)/C=C/C=C(/C)C(=O)NC</smiles>
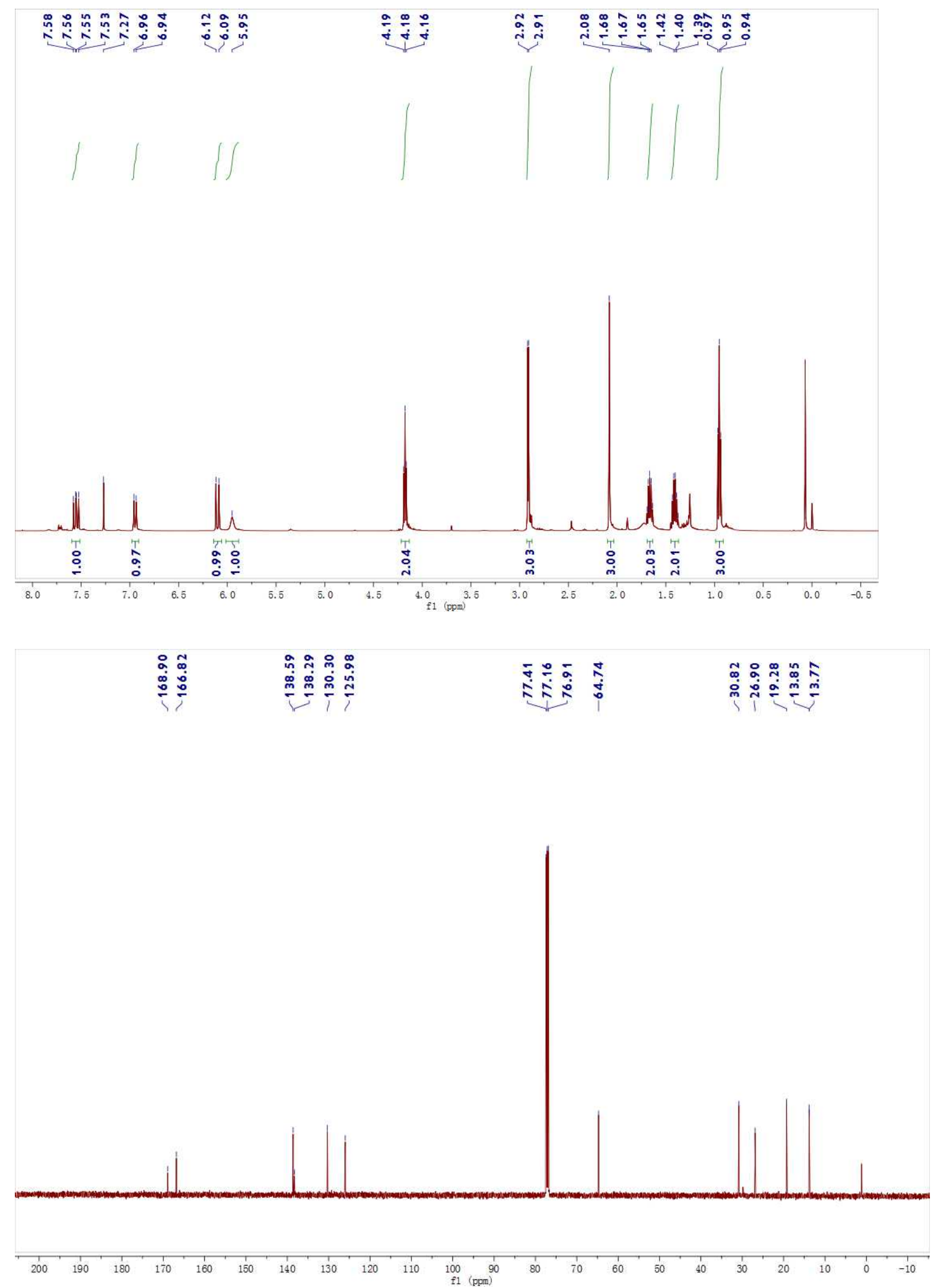
<smiles>CCCCC(=O)OCCCC(C)C(=O)N(C)C</smiles>

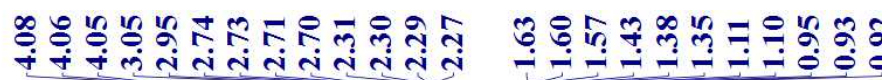

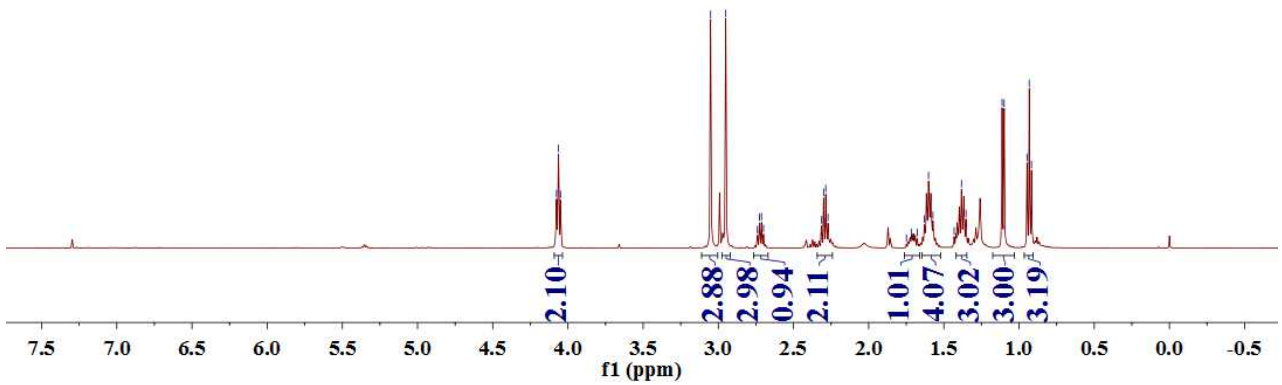

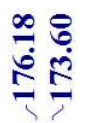

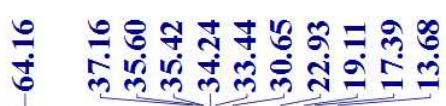

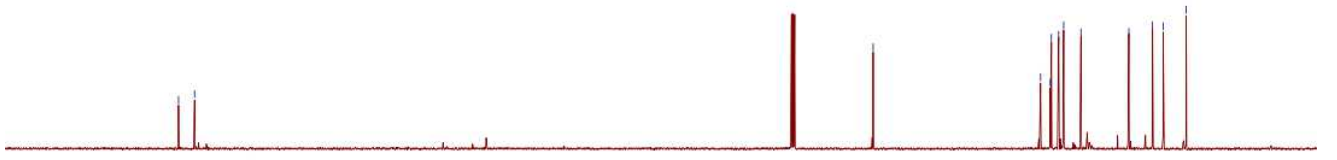

$\begin{array}{lllllllllllllllllllll}200 & 190 & 180 & 170 & 160 & 150 & 140 & 130 & 120 & 110 & \begin{array}{c}100 \\ \mathrm{f} 1(\mathrm{ppm})\end{array} & 90 & 80 & 70 & 60 & 50 & 40 & 30 & 20 & 10 & 0\end{array}$ 
$\mathrm{MeO}^{\mathrm{M}}{ }^{\mathrm{N}}$
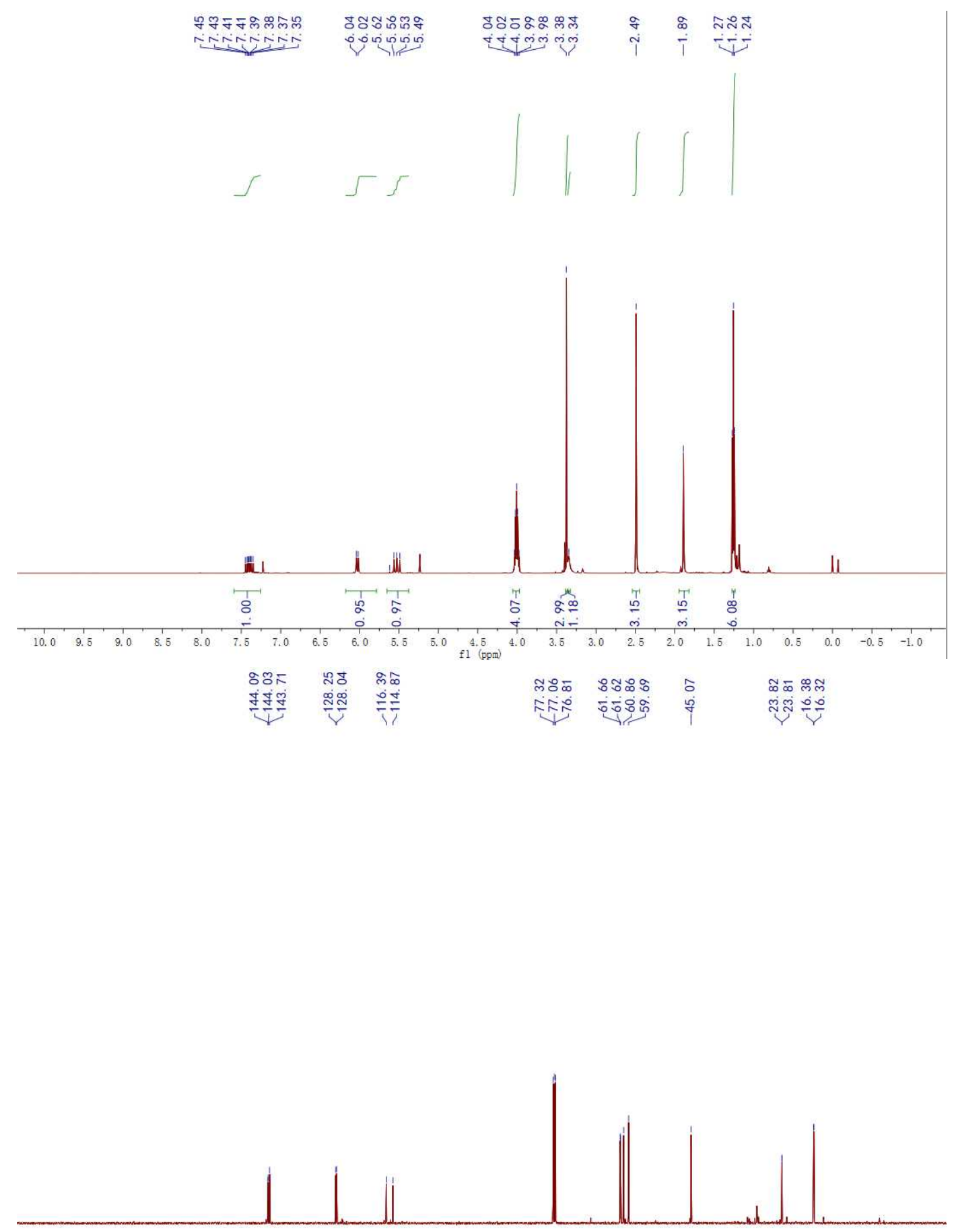

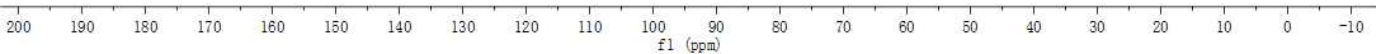



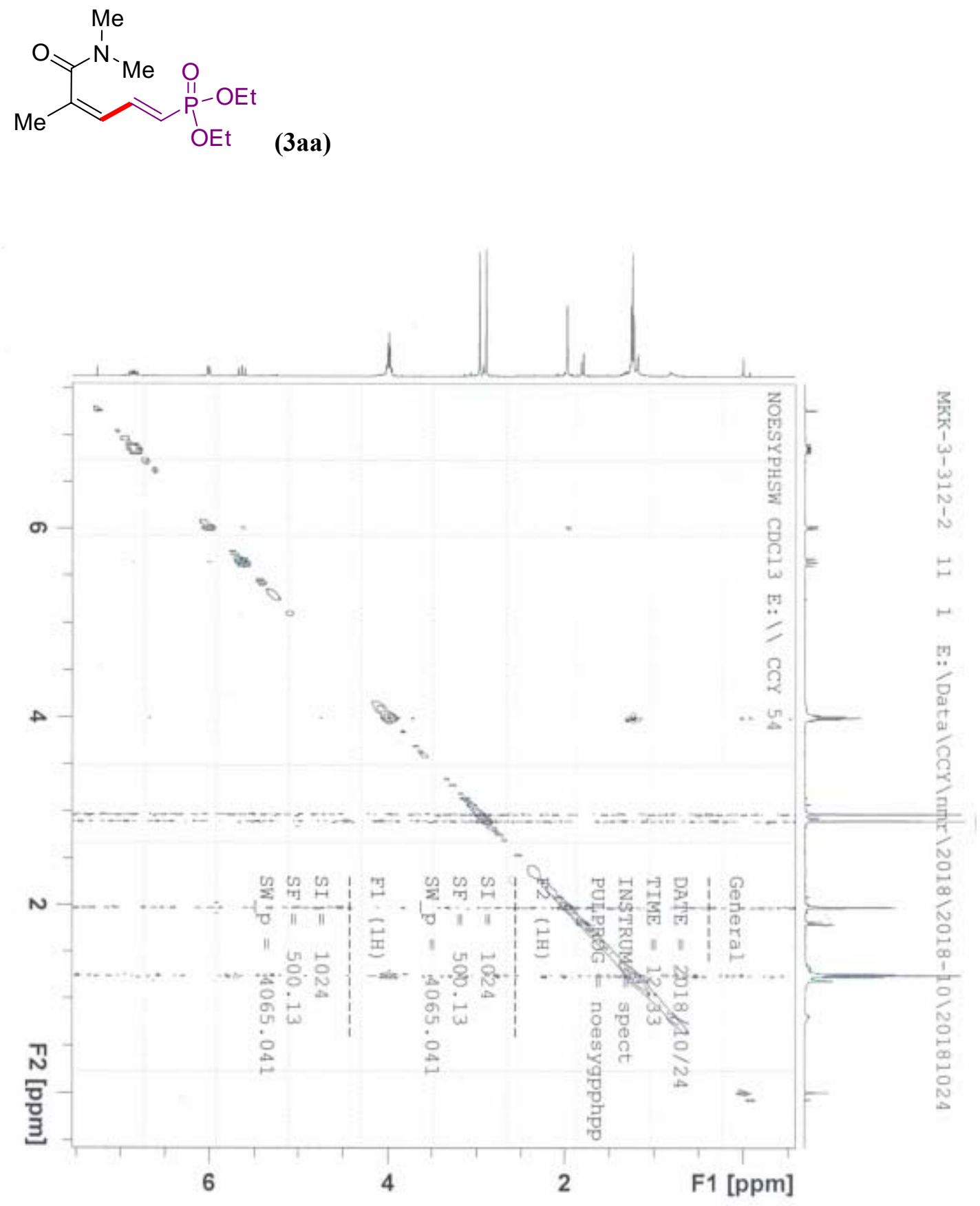